\title{
Decay of shocklike waves into compact oscillons
}

\author{
F. M. Hahne®, P. Klimas@, and J. S. Streibel® \\ Departamento de Física, Universidade Federal de Santa Catarina, \\ Campus Trindade, 88040-900 Florianópolis-SC, Brazil
}

(Received 13 December 2019; accepted 3 April 2020; published 16 April 2020)

\begin{abstract}
The signum-Gordon model in $1+1$ dimensions possesses an exact solution with a discontinuity of the field at the light cone. Thus, the gradient energy of these waves is infinite whereas the energy of a part of the wave localized inside the light cone is finite and grows linearly with time. The initial data for such waves contain a field configuration which is null in space and its time derivative is proportional to the Dirac delta. We study regular initial data that lead to shocklike waves with finite gradient energy. We found that such waves exist only for a finite time and eventually decay in a cascade of oscillonlike structures. The pattern of decay is very similar to the one observed in the scattering of compact oscillons.
\end{abstract}

DOI: 10.1103/PhysRevD.101.076013

\section{INTRODUCTION}

Compact oscillons [1] in the signum-Gordon model [2] are rather unusual field configurations. They do not match well any of the two groups containing qualitatively different types of oscillating (quasi)periodic excitations of scalar fields-oscillons and breathers. Whereas oscillons [3-8] are quasiperiodic and slowly radiating excitations in nonintegrable field theories, the breathers [9-12] are exact infinitely long living solutions in many integrable models. In this sense, the presence or lack of emission of the radiation from such periodic structures indicates the model may or may not be integrable. The signum-Gordon oscillon is an exact, compact, perfectly periodic and finite energy solution. Although it does not radiate at all, when perturbed it emits some radiation [13]. Since the signum-Gordon model is a nonintegrable field theory, the existence of infinitely long living exact oscillons is a rather unusual phenomenon. Such oscillons may have wider applications in theories with approximate scaling symmetry [14]. It is possible due to the fact that the signum-Gordon model emerges from many field theories with nondifferentiable potentials in the limit of small amplitude excitations [1316]. Such more general models can support topological solitons and defects [17]. An interesting problem associated with the presence of oscillons is their production during scattering of kinks, skyrmions and other topological or nontopological objects. Quite recently we have looked at the process of scattering of the signum-Gordon oscillons

Published by the American Physical Society under the terms of the Creative Commons Attribution 4.0 International license. Further distribution of this work must maintain attribution to the author(s) and the published article's title, journal citation, and DOI. Funded by SCOAP . and production of a radiation being dominated by smaller oscillons [18]. Motivated by this study we shall look in more detail at the process of the collapse of signum-Gordon shock waves which is an efficient process of emission of oscillons. This is the subject of the present paper. We expect that similar phenomena may be observed in models with approximate scaling symmetry in the limit of small amplitudes. Our study may have applications in such models and can contribute to a better understanding of the dynamics of small amplitude oscillations of fields close to the vacua $[14,19]$.

The signum-Gordon model is a scalar field theory with a self-interaction term which is proportional to $\phi /|\phi|$. Such a term corresponds with the potential $V=|\phi|$ which is a particular case of a wider group of the so-called $\mathrm{V}$-shaped potentials. The signum-Gordon model describes a universal behavior of the field in the vicinity of $\mathrm{V}$-shaped minima in a similar way as the Klein-Gordon equation describes dynamics of small amplitude oscillations of fields in the model with parabolic potentials around minima. According to Ref. [14], a symmetry reduction in certain physical models results in limitations on values taken by new fields and, consequently, in the appearance of models with $\mathrm{V}$-shaped potentials. Therefore, the solutions of the signum-Gordon equation,

$$
\left(\partial_{t}^{2}-\nabla^{2}\right) \phi(t, \vec{x})+\operatorname{sgn} \phi(t, \vec{x})=0,
$$

have universal character in the sense that they can appear as solutions (or approximations of true solutions) of many other models. For instance, the signum-Gordon oscillons were found in the second Bogomol'nyi-Prasad-Sommerfield (BPS) submodel of the Skyrme model [13,14].

The signum-Gordon model is well behaving from a physical viewpoint and, in particular, it admits a mechanical 
realization. The potential $V=|\phi|$ is fundamentally nonlinear only at $\phi=0$. Thus the signum-Gordon model shares some aspects of linear and nonlinear field theories. The nonlinear character is associated with the minimum of the potential; i.e., it is particularly visible for small amplitude fields. Contrary to many other nonlinear field theories it cannot be linearized in the limit $\phi \rightarrow 0$, which means that small amplitude perturbations are always nonlinear. On the other hand, in the regions of space where the sign of the field is fixed, the signum-Gordon equation reduces to a nonhomogeneous linear wave equation. In this paper we shall look at the real valued model in one spatial dimension. It means that $\nabla^{2} \rightarrow \partial_{x}^{2}$. In such a case there exists a general expression for solutions with a fixed sign, $\operatorname{sgn} \phi_{k}= \pm 1$,

$$
\phi_{k}(t, x)=F_{k}(x+t)+G_{k}(x-t) \pm \frac{1}{4}\left(x^{2}-t^{2}\right),
$$

where $F_{k}(z)$ and $G_{k}(z)$ are some arbitrary functions. Such solutions are called partial solutions and each of them has a domain corresponding with a support ${ }^{1}$ labeled by $k$. A physical solution consists on a certain number (usually infinitely many) of properly matched partial solutions. Thus, linearity is rather a local property associated with individual partial solutions and it cannot be extended on solutions of the model.

The signum-Gordon model is certainly nonintegrable. However, it possesses a quite large family of exact solutions, for instance self-similar solutions [20,21], exact oscillons [1,22,23], and shock waves [20,24]. This fact is related to the existence of a quite general expression for partial solutions (1.2). In this paper we study solutions which are closely related with shock waves. The motivation for such a study came from the analysis of recent results for scattering of the signum-Gordon oscillons [18], where shock-wave-like configurations form right after oscillon interaction and then break and decay into a cascade of oscillons.

The paper is organized as follows. In Sec. II, we give a short revision of shock waves in the signum-Gordon model and present initial conditions which lead to such solutions. In Sec. III, we discuss the problem of energy density and total energy of shock waves. Section IV is devoted to the presentation of numerical and analytical solutions obtained from the initial configuration of the field which contains a deltalike initial profile of the time derivative.

\section{THE SIGNUM-GORDON SHOCK WAVES}

\section{A. The solution}

The shock waves form a particular class of exact solutions of the signum-Gordon model that stand out against its other solutions by the presence of a discontinuity

\footnotetext{
${ }^{1}$ Usually this support is compact.
}

of the field at the light cone. This class of solutions has been proposed in Ref. [20]. It is obtained by the reduction of the signum-Gordon equation to an ordinary differential equation via ansatz

$$
\phi(t, x)=\theta(-z) W(z), \quad \text { where } z=\frac{1}{4}\left(x^{2}-t^{2}\right),
$$

where $\theta(-z)$ is the Heaviside step function. The function $W(z)$ obeys the equation

$$
z W^{\prime \prime}(z)+W^{\prime}(z)=\operatorname{sgn}(W(z))
$$

and it consists of infinitely many partial solutions $W_{k}(z)$, $k \in \mathbb{Z}$ matched at points $-a_{k}$. Each partial solution satisfies the equation

$$
z W_{k}^{\prime \prime}(z)+W_{k}^{\prime}(z)=(-1)^{k}
$$

and the matching conditions $W_{k}\left(-a_{k}\right)=0=W_{k+1}\left(-a_{k}\right)$ and $W_{k}^{\prime}\left(-a_{k}\right)=W_{k+1}^{\prime}\left(-a_{k}\right)$. Imposing the condition $W_{k}\left(-a_{k}\right)=0$ we get the partial solutions in the form

$$
W_{k}(z)=(-1)^{k}\left(z+a_{k}+b_{k} \ln \frac{|z|}{a_{k}}\right) .
$$

Then, imposing the remaining conditions we get some restrictions on the coefficients $a_{k}$ and $b_{k}$, namely

$\frac{b_{k+1}}{a_{k}}=2-\frac{b_{k}}{a_{k}}, \quad$ and $\quad \frac{a_{k+1}}{a_{k}}=1+\frac{b_{k+1}}{a_{k}} \ln \frac{a_{k+1}}{a_{k}}$.

Note that $b_{0}$ must vanish in order to avoid the singularity of the logarithm at $z=0$. The first zero $a_{0}$ can be chosen as a free parameter which, in turn, determines the values of all other constants via recurrence relations (2.3). In particular, it holds $b_{1}=2 a_{0}$. In terms of new variables $\alpha_{k+1}:=\frac{1}{2} \frac{b_{k+1}}{a_{k}}$ and $y_{k+1}:=\frac{a_{k+1}}{a_{k}}$, the relations (2.3) take the form

$\alpha_{k+1}=1-\frac{\alpha_{k}}{y_{k}}, \quad$ and $\quad y_{k+1}=1+2 \alpha_{k+1} \ln y_{k+1}$,

where $\alpha_{1}=1$. It follows from (2.4) that $\alpha_{k+1}$ is determined by $a_{k}$ and $b_{k}$. Then, solving numerically the second equation of (2.4) one gets $y_{k+1}$ and thus both coefficients $a_{k+1}$ and $b_{k+1}$ can be determined. We shall skip the discussion of the solution of the recurrence relations because it is given in previous papers. In Fig. 1(a) we present the first few partial solutions $W_{k}(z)$ and in Fig. 1(b) we present the shock wave at $t=6.5$, which consists of four partial solutions.

In Fig. 2 we show the shock wave on the Minkowski diagram. The zeros of the field $\phi(t, x)$ correspond with hyperbolas 


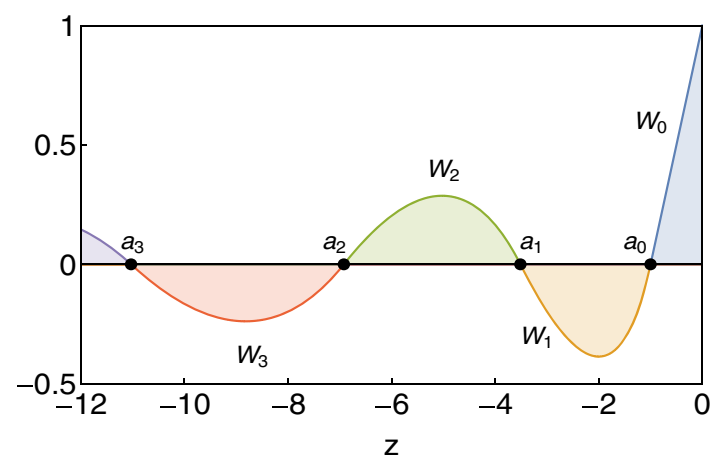

(a) $W_{k}(x)$

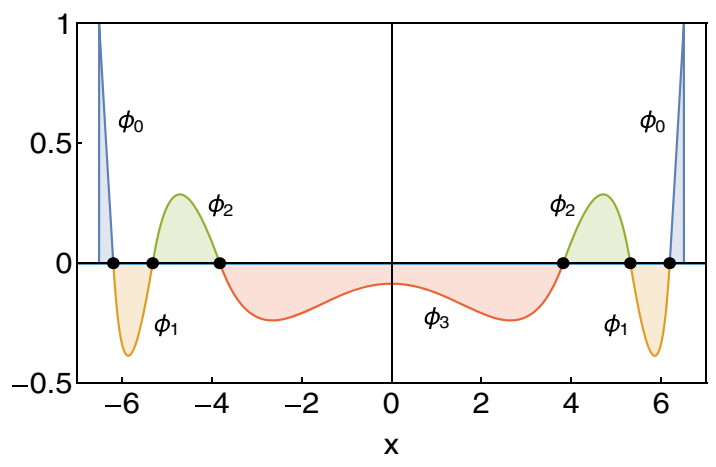

(b) $\phi_{k}(t=6.5, x)$

FIG. 1. The exact shock wave solution for $a_{0}=1$.

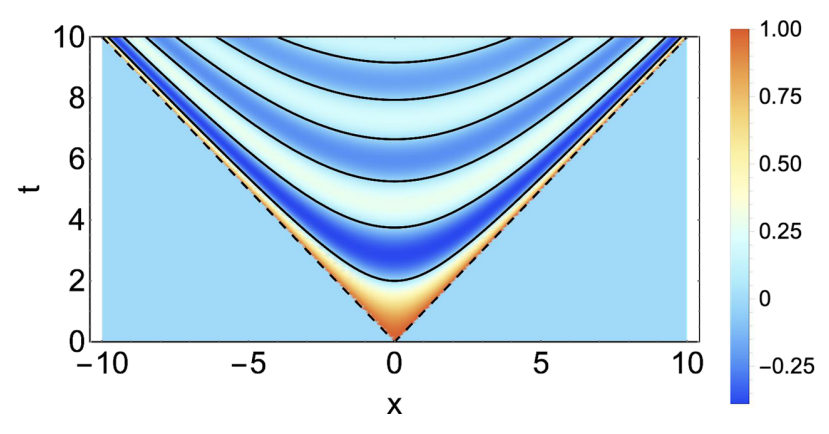

FIG. 2. The exact shock wave solution for $a_{0}=1$ at the spacetime diagram.

$$
x_{k}(t)= \pm \sqrt{t^{2}-4 a_{k}}
$$

In contrast to the case of compact oscillons or self-similar solutions, there is very little known about the role of shock waves in the dynamics of more complex field configurations in the signum-Gordon model. We have not seen such solutions in numerical simulations of interaction between kinks and perturbations of exact oscillons. However, this situation has changed after getting the first results of the scattering of high speed oscillons; see Ref. [18]. We have found that such oscillons produce radiation that mainly consists of perturbed oscillons. The oscillons do not appear immediately after the collision. The numerical study shows that they are generated in the process of breaking of the wave field configuration. We have confirmed that these waves are related to shock waves by comparing their zeros with the zeros of exact shock waves. It raised the question about initial conditions that support such shock-wave-like configurations. We shall answer this question in the subsequent section.

\section{B. The initial problem}

An important question we would like to answer now is a relation between the discontinuity of the field at the light cone and the initial conditions which would produce such a discontinuity. This problem has not been answered in the original papers which address the construction of shock waves in the signum-Gordon model and its modifications. The analysis of the shock wave solution allows one to conclude that in the limit $t \rightarrow 0+$, the scalar field $\phi$ and its first derivative $\partial_{t} \phi$ vanish everywhere except the point $x=0$. It suggests the possible choice of initial configuration of the field $\phi(0, x)=0$ everywhere and $\left.\partial_{t} \phi(t, x)\right|_{t=0}$ proportional to the Dirac delta $\delta(x)$. The problem of the discontinuity of the field at the light cone can be studied without the necessity of solving the complete signumGordon equation.

\section{The initial problem for the wave equation}

Looking at the problem of the appearance of discontinuities of the field, it is enough to look at a simpler problem involving the wave equation in $(1+1)$ dimensions $\left(\partial_{t}^{2}-\partial_{x}^{2}\right) \phi(t, x)=0$. There is another advantage associated with this simplification-we have a general solution of the initial problem given by the d'Alembert formula. Let $\phi(0, x)=f(x)$ and $\left.\partial_{t} \phi(t, x)\right|_{t=0}=g(x)$ be an initial field configuration, then the wave equation in one spatial dimension has its solution given by

$\phi(t, x)=\frac{1}{2}(f(x+t)+f(x-t))+\frac{1}{2} \int_{x-t}^{x+t} d \xi g(\xi)$.

Taking particular initial conditions which are suitable for our considerations

$\phi(0, x)=f(x)=0,\left.\quad \partial_{t} \phi(t, x)\right|_{t=0}=g(x)=a \delta(x)$,

where $a=$ const, we find

$$
\phi(t, x)=\frac{a}{2}(\theta(x+t)-\theta(x-t))= \begin{cases}\frac{a}{2} & \text { for }|x|<t \\ 0 & \text { for }|x|>t\end{cases}
$$

Thus the solution $\phi(t, x)$ is equal to $a / 2$ inside the light cone and it vanishes outside the light cone. Clearly, the field 


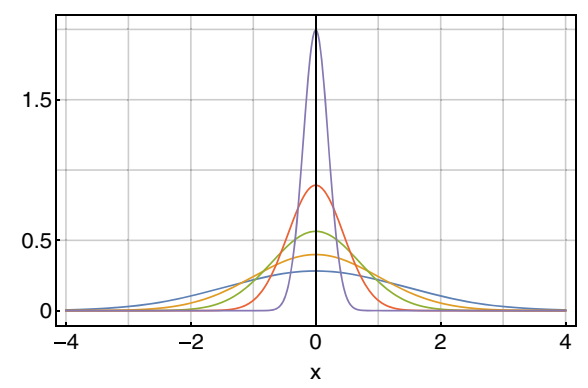

(a) $\delta_{\epsilon}(x)-$ Gaussian

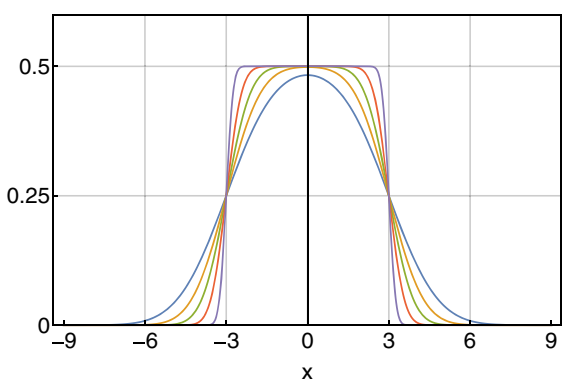

(b) $\phi(t=3, x), \quad a=1$

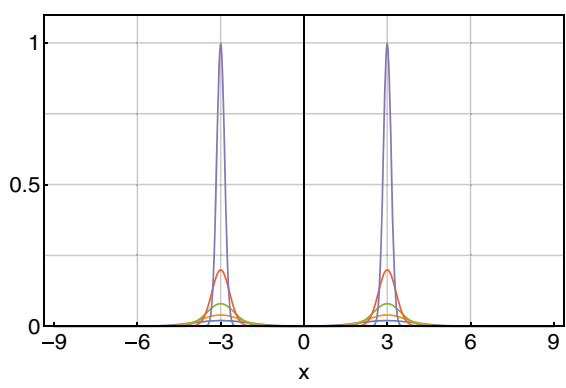

(c) $u(t=3, x), \quad a=1$

FIG. 3. (a) Function $\delta_{\epsilon}(x)$ and (b) solution $\phi(t, x)$ and (c) energy density for $\epsilon=\left\{1, \frac{1}{2}, \frac{1}{4}, \frac{1}{10}, \frac{1}{50}\right\}$.

possesses discontinuity at the light cone $x= \pm t$. The same behavior at the light cone is expected for the signumGordon equation. However, in the signum-Gordon model the sign term modifies the solution inside the light cone, which leads to observed oscillations.

\section{Regular initial data}

The presence of a discontinuity in the field has some unpleasant consequences. This is pretty clear when looking at the problem of the energy associated with such field configurations. To see it, we look again at the problem restricted to the solutions of the wave equation in one spatial dimension. The energy of a solution is given by the expression

$$
E=\frac{1}{2} \int_{-\infty}^{\infty} d x\left(\left(\partial_{t} \phi\right)^{2}+\left(\partial_{x} \phi\right)^{2}\right)
$$

Looking at partial derivatives of the solution (2.8) one gets

$$
\begin{aligned}
& \partial_{t} \phi(t, x)=\frac{a}{2}(\delta(x+t)+\delta(x-t)), \\
& \partial_{x} \phi(t, x)=\frac{a}{2}(\delta(x+t)-\delta(x-t)) .
\end{aligned}
$$

It raises the problem about the expression " $(\delta(x))^{2}$ ", which is meaningless as a distribution. Thus we see that this question is related to the problem of "multiplication of generalized functions".

In order to overcome this problem we shall replace the delta distribution $\delta(x)$ in (2.7) by a classical function. There are many classical functions $\delta_{\epsilon}(x)$ which tend to the Dirac delta when $\epsilon \rightarrow 0$. This limit has the following sense:

$$
\lim _{\epsilon \rightarrow 0} \int_{-\infty}^{\infty} d x \delta_{\epsilon}(x) \varphi(x)=\varphi(0) \equiv \int_{-\infty}^{\infty} d x \delta(x) \varphi(x)
$$

where $\varphi(x)$ is a test function.

We shall consider two such functions in our analysis, namely a Gaussian function,

$$
\delta_{\epsilon}(x)=\frac{1}{2 \sqrt{\pi \epsilon}} e^{-\frac{x^{2}}{4 \epsilon}}
$$

and a triangular function with compact support,

$$
\begin{aligned}
\delta_{\epsilon}(x)= & \frac{1}{4 \pi \epsilon}[(2 \sqrt{\pi \epsilon}+x) \theta(2 \sqrt{\pi \epsilon}+x) \theta(-x) \\
& +(2 \sqrt{\pi \epsilon}-x) \theta(2 \sqrt{\pi \epsilon}-x) \theta(x)] .
\end{aligned}
$$

We have chosen two different classes of functions $\delta_{\epsilon}$ in order to see to which extent the solutions of the wave equation depend on the choice. Functions (2.12) and (2.13) satisfy

$$
\int_{-\infty}^{\infty} d x \delta_{\epsilon}(x)=1, \quad \delta_{\epsilon}(0)=\frac{1}{2 \sqrt{\pi \epsilon}}
$$

The triangular function $\delta_{\epsilon}$ has been normalized in a way that it takes the same value at the center $x=0$ as the Gaussian function. The plots corresponding with (2.12) are shown in Fig. 3(a) and those corresponding with (2.13) in Fig. 4(a).

The d'Alembert formula (2.6) allows one to obtain an exact solution for $f(x)=0$ and $g(x)=a \delta_{\epsilon}(x)$. When $\delta_{\epsilon}$ is a Gaussian function one gets the expression

$$
\phi(t, x)=\frac{a}{4}\left(\operatorname{erf}\left[\frac{x+t}{2 \sqrt{\epsilon}}\right]-\operatorname{erf}\left[\frac{x-t}{2 \sqrt{\epsilon}}\right]\right)
$$

where $\operatorname{erf}(z)$ stands for the error function. ${ }^{2}$ Figure 3(b) shows a few solutions $\phi(t, x)$ at $t=3$ for different values of the parameter $\epsilon$. In the limit $\epsilon \rightarrow 0$, the solution (2.14) tends to (2.8). In the case of initial data given by (2.13), one can also obtain an exact solution. However, this solution is technically more complex because it contains different expressions in different regions of spacetime. The partial

$$
\operatorname{erf}(z):=\frac{2}{\sqrt{\pi}} \int_{0}^{z} d u e^{-u^{2}}=\frac{2}{\sqrt{\pi}} \sum_{n=0}^{\infty} \frac{(-1)^{n} z^{2 n+1}}{n !(2 n+1)}
$$




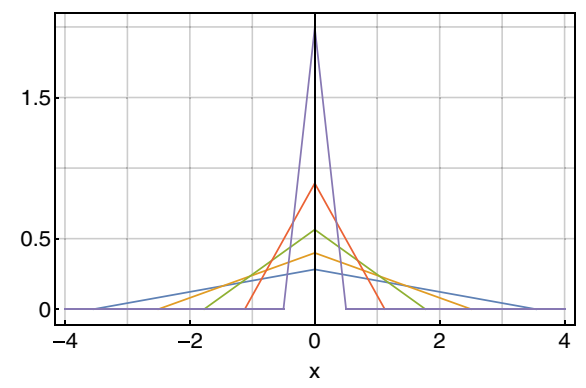

(a) $\delta_{\epsilon}(x)$ - triangular

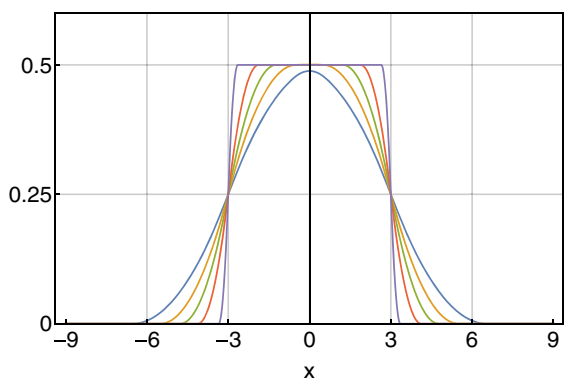

(b) $\phi(t=3, x), \quad a=1$

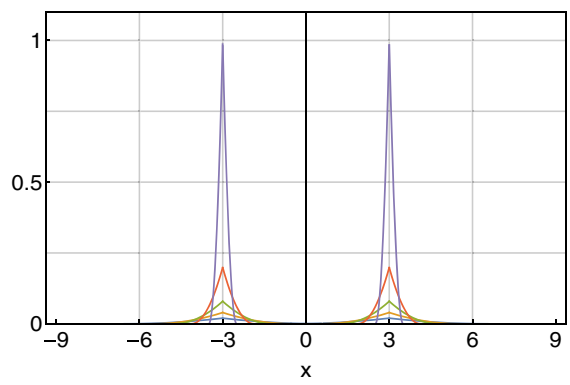

(c) $u(t=3, x), \quad a=1$

FIG. 4. (a) Function $\delta_{\epsilon}(x)$ and (b) solution $\phi(t, x)$ and (c) energy density for $\epsilon=\left\{1, \frac{1}{2}, \frac{1}{4}, \frac{1}{10}, \frac{1}{50}\right\}$.

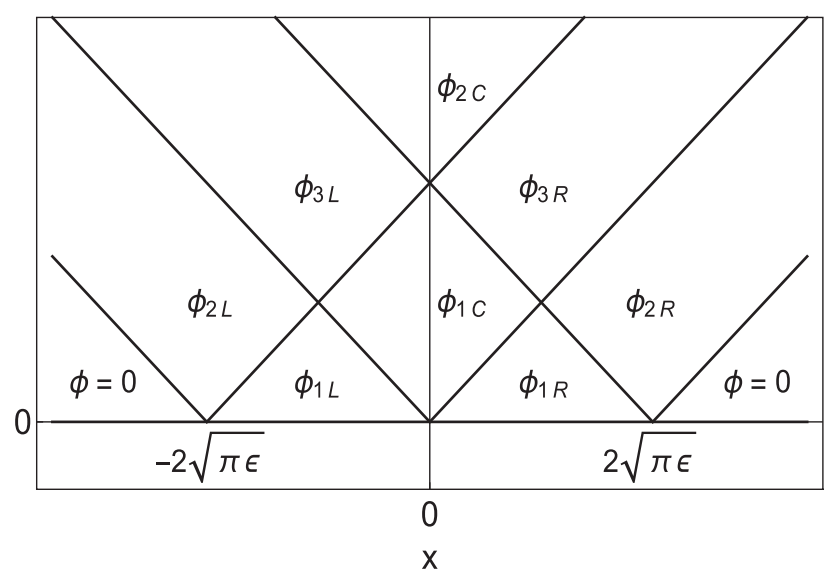

FIG. 5. Partial solutions for the triangle-shape initial profile of $\partial_{t} \phi$.

solutions vanish outside their supports. In Fig. 5 we show the regions where the supports of partial solutions are localized. The partial solutions that form the solution of the problem are given by the expressions

$$
\begin{gathered}
\phi_{1 L}(t, x)=\frac{a}{4 \pi \epsilon}(2 \sqrt{\pi \epsilon}+x) t, \\
\phi_{2 L}(t, x)=\frac{a}{4 \pi \epsilon}\left(\frac{x+t}{2}+\sqrt{\pi \epsilon}\right)^{2}, \\
\phi_{3 L}(t, x)=\frac{a}{4 \pi \epsilon}\left(\pi \epsilon+\sqrt{\pi \epsilon}(x+t)-\frac{(x+t)^{2}}{4}\right), \\
\phi_{1 C}(t, x)=\frac{a}{4 \pi \epsilon}\left(2 \sqrt{\pi \epsilon} t-\frac{x^{2}+t^{2}}{2}\right), \\
\phi_{2 C}(t, x)=\frac{a}{2}, \\
\phi_{k R}(t, x)=\phi_{k L}(t,-x) \quad \text { for } k=1,2,3 .
\end{gathered}
$$

Note that all expressions presented above are given by polynomials of degree no higher than two. Figure 4(b) shows the profile of the solution $\phi(t, x)$ at $t=3$ for various values of $\epsilon$. In the case of initial data given by the triangle shape of the initial profile of $\partial_{t} \phi$, the solution is compact.

Finally, one can look at the energy density and total energy of such solutions. Since the solution $\phi(t, x)$ is given by classical functions then there is no problem with taking the square of its derivatives. In the case of the solution (2.14) obtained for Gaussian initial data, one gets the following expression for the total energy of the field:

$$
\begin{aligned}
E & =\int_{-\infty}^{\infty} d x u(t, x)=\frac{a^{2}}{16 \pi \epsilon} \int_{-\infty}^{\infty} d x\left(1+e^{\frac{2 t x}{\epsilon}}\right) e^{-\frac{(x+t)^{2}}{2 \epsilon}} \\
& =\frac{a^{2}}{4 \sqrt{2 \pi \epsilon}}
\end{aligned}
$$

The energy density shown in Fig. 3(c) is finite everywhere. Also, the total energy of the field is finite. However, it depends on $\epsilon$. In the limit $\epsilon \rightarrow 0$ the energy tends to infinity as $\epsilon^{-1 / 2}$ is what is associated with the formation of discontinuities of the field and the increasing of the gradient energy.

In the case of triangle-shape profile (2.13) we find that the energy density is a finite function that consists on the patches

$$
\begin{aligned}
& u_{1 L}(t, x)=\frac{a^{2}}{32 \pi^{2} \epsilon^{2}}\left[t^{2}+(x+2 \sqrt{\pi \epsilon})^{2}\right], \\
& u_{2 L}(t, x)=\frac{a^{2}}{64 \pi^{2} \epsilon^{2}}[t+x+2 \sqrt{\pi \epsilon}]^{2}, \\
& u_{3 L}(t, x)=\frac{a^{2}}{64 \pi^{2} \epsilon^{2}}[t+x-2 \sqrt{\pi \epsilon}]^{2}, \\
& u_{1 C}(t, x)=\frac{a^{2}}{32 \pi^{2} \epsilon^{2}}\left[x^{2}+(t-2 \sqrt{\pi \epsilon})^{2}\right], \\
& u_{2 C}(t, x)=0, \\
& u_{k R}(t, x)=u_{k L}(t,-x) \quad \text { where } k=1,2,3 .
\end{aligned}
$$

The plot of this energy density is shown in Fig. 4(c). In similarity to the Gaussian case the energy density is a finite function for $\epsilon \neq 0$. The only difference is that now the 
energy density vanishes explicitly outside the two compact supports localized around the light cone of the event $(0,0)$. The total energy in this case reads

$$
E=\int_{-2 \sqrt{\pi \epsilon}}^{2 \sqrt{\pi \epsilon}} d x u(t, x)=\frac{a^{2}}{6 \sqrt{\pi \epsilon}}
$$

It has exactly the same functional behavior as for the Gaussian case.

\section{THE ENERGY OF A SHOCK WAVE}

A fundamental difference between the wave equation and the signum-Gordon equation is the presence of the selfinteraction described by the potential $V(\phi)=|\phi|$, which gives rise to the term $\operatorname{sgn}(\phi)$ in the field equation. It means that each nonvanishing field configuration has also some potential energy. Consequently, the wave on an open segment $-t<x<t$ has some finite energy. This energy consists on three elements: kinetic, gradient, and potential energy. Of course, similarly to the solution of the d'Alembert equation, there is also an infinite gradient energy due to discontinuities at $x= \pm t$. The gradient energy stored in the discontinuity of the field at the light cone is essential for the existence of shock waves. The discontinuities of the field constitute a sort of energy reservoir feeding the regular wave in the central region inside the future light cone of the event $(0,0)$. In order to make this statement clearer, we look in more detail to the wave on an open segment $-t<x<t$, especially to the energies of its partial solutions.

A general solution describing a shock wave is a sum ${ }^{3}$ of partial solutions $\phi_{k}(t, x)=W_{k}(z)$

$$
\phi(t, x)=\sum_{k=0}^{\infty} \phi_{k}(t, x),
$$

where $W_{k}(z) \equiv 0$ outside the support $a_{k} \leq z \leq a_{k-1}$. At the instant of time

$$
t_{k-1}=2 \sqrt{a_{k-1}},
$$

the partial solution $\phi_{k-1}$ splits into two disjointed parts and there appears the solution $\phi_{k}(t, x)$. The solution $\phi_{k}(t, x)$ exists on the support

$$
\operatorname{supp}_{\phi_{k}}(t):= \begin{cases}|x| \leq c_{k-1}(t) & \text { for } t_{k-1} \leq t \leq t_{k} \\ c_{k}(t) \leq|x| \leq c_{k-1}(t) & \text { for } t \geq t_{k}\end{cases}
$$

where

\footnotetext{
${ }^{3}$ Each partial solution vanishes outside its own support.
}

TABLE I. Zeros $a_{k}$ of $W(z)$ and instants of time $t_{k}=2 \sqrt{a_{k}}$ at which each solution $\phi_{k+1}(t, x)$ appears.

\begin{tabular}{lcccrrrr}
\hline \hline & $k=0$ & $k=1$ & $k=2$ & $k=3$ & $k=4$ & $k=5$ & $k=6$ \\
\hline$a_{k}$ & 1.00 & 3.51 & 6.92 & 11.03 & 15.73 & 20.96 & 26.67 \\
$t_{k}$ & 2.00 & 3.75 & 5.26 & 6.64 & 7.93 & 9.16 & 10.33 \\
\hline \hline
\end{tabular}

$$
c_{k}(t):=\sqrt{t^{2}-4 a_{k}}
$$

has an interpretation of positive zeros of the wave. The values of $a_{k}$ and $t_{k}$ for $a_{0}=1$ are given in Table I. The function (3.1) is a $\mathcal{C}^{1}$ class at $x \in(-t, t)$.

The energy associated with the wave on the open segment $-t<x<t$ is given by

$$
E(t)=\sum_{k=0}^{N(t)}\left(K_{k}(t)+U_{k}(t)\right)
$$

where $N(t)+1$ is the number of partial solutions at $t$ that form a shockwave, and

$$
\begin{aligned}
K_{k}(t) & :=\frac{1}{2} \int_{-t}^{t} d x\left[\left(\partial_{t} \phi_{k}\right)^{2}+\left(\partial_{x} \phi_{k}\right)^{2}\right] \\
& =\int_{\operatorname{supp}_{\phi_{k}}(t)} d x\left[\frac{x^{2}+t^{2}}{8}\left(W_{k}^{\prime}\left(\frac{x^{2}-t^{2}}{4}\right)\right)^{2}\right], \\
U_{k}(t) & :=\int_{-t}^{t}\left|\phi_{k}\right| d x=\int_{\operatorname{supp}_{\phi_{k}}(t)} d x\left|W_{k}\left(\frac{x^{2}-t^{2}}{4}\right)\right|
\end{aligned}
$$

describe contributions of individual partial solutions to the energy of the wave inside the light cone. In Fig. 6 we show integrands (densities) of (3.6) and (3.7) at $t=t_{6}$.

Both integrals presented above can be given by explicit functions. First we shall look at the contributions to kinetic and gradient energy. We shall present separately the cases $k=0$ and $k=1,2,3, \ldots$ In the case $k=0$, we get $W_{0}^{\prime}=1$. Then

$K_{0}(t)=2 \int_{x_{\min }(t)}^{x_{\max }(t)} d x \frac{x^{2}+t^{2}}{8}=\frac{1}{4}\left[t^{2} x-\frac{x^{3}}{3}\right]_{x_{\min }(t)}^{x_{\max }(t)}$,

where the factor " 2 " appears due to the spatial symmetry of the wave, and

$$
\left(x_{\min }, x_{\max }\right)=\left\{\begin{array}{ll}
(0, t) & \text { for } \quad 0 \leq t \leq t_{0} \\
\left(c_{0}(t), t\right) & \text { for } t \geq t_{0}
\end{array},\right.
$$

where $t_{0}$ is given by (3.2). The integral (3.8) reads 


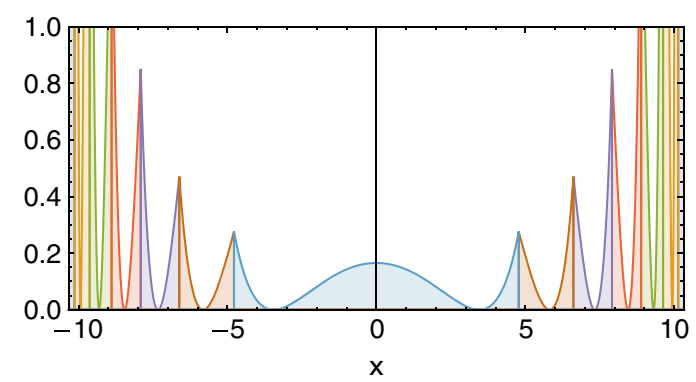

(a) $\frac{1}{2}\left(\partial_{t} \phi_{k}\right)^{2}+\frac{1}{2}\left(\partial_{x} \phi_{k}\right)^{2}, \quad k=0,1, \ldots, 6$

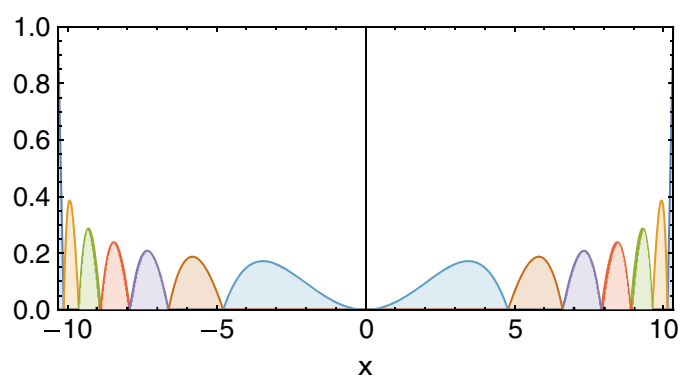

(b) $\left|\phi_{k}(t, x)\right|, \quad k=0,1, \ldots, 6$

FIG. 6. (a) Density of kinetic and gradient energy and (b) density of potential energy associated with the wave at $t=t_{6}$.

$$
K_{0}(t)=\left\{\begin{array}{ll}
\frac{t^{3}}{3} & \text { for } 0 \leq t \leq t_{0} \\
\frac{t^{3}}{3}-\frac{1}{3}\left(t^{2}-a_{0}\right) c_{0}(t) & \text { for } t \geq t_{0}
\end{array} .\right.
$$

The expression $K_{0}(t)$ in the limit of large $t$ has linear behavior

$$
K_{0}(t) \approx a_{0} t+\mathcal{O}\left(t^{-3}\right)
$$

The energies $K_{k}(t)$ with $k=1,2, \ldots$ are given by the expression

$$
K_{k}(t)=2 \int_{x_{\min }(t)}^{x_{\max }(t)} d x \frac{x^{2}+t^{2}}{8}\left(1+\frac{4 b_{k}}{x^{2}-t^{2}}\right)^{2}
$$

where

$$
\left(x_{\min }, x_{\max }\right)=\left\{\begin{array}{ll}
\left(0, c_{k-1}(t)\right) & \text { for } t_{k-1} \leq t \leq t_{k} \\
\left(c_{k}(t), c_{k-1}(t)\right) & \text { for } t \geq t_{k}
\end{array},\right.
$$

and where $t_{k}$ and $c_{k}$ are given by (3.2) and (3.4). The kinetic and gradient part of the energy reads

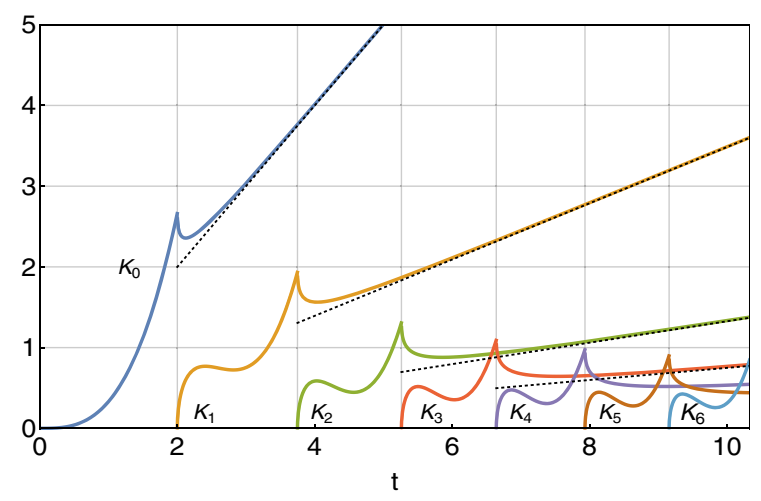

(a) $K_{k}(t)$
$K_{k}(t)=\left\{\begin{array}{ll}K_{k}^{(-)}(t)=P_{k}(t) & \text { for } \quad t_{k-1} \leq t \leq t_{k} \\ K_{k}^{(+)}(t)=P_{k}(t)+Q_{k}(t) & \text { for } t \geq t_{k}\end{array}\right.$,

where we have defined the symbols

$$
\begin{aligned}
P_{k}(t):= & {\left[\frac{b_{k}^{2}}{a_{k-1}}+2 b_{k}-\frac{a_{k-1}}{3}+\frac{t^{2}}{3}\right] c_{k-1}(t) } \\
& -4 b_{k} t \operatorname{ArcTanh}\left(\frac{c_{k-1}(t)}{t}\right), \\
Q_{k}(t):= & -\left[\frac{b_{k}^{2}}{a_{k-1}}+2 b_{k}-\frac{a_{k}}{3}+\frac{t^{2}}{3}\right] c_{k}(t) \\
& -4 b_{k} t \operatorname{ArcTanh}\left(\frac{c_{k}(t)}{t}\right) .
\end{aligned}
$$

The expression $K_{k}(t)$ has the following asymptotic behavior for $t \rightarrow \infty$ :

$$
K_{k}(t) \approx\left[\frac{b_{k}^{2}}{a_{k-1}}-\frac{b_{k}^{2}}{a_{k}}+a_{k-1}-a_{k}\right] t+\mathcal{O}\left(t^{-3}\right) .
$$

In Fig. 7(a) we show the first seven contributions $K_{k}(t)$ associated with derivative terms in the energy density.

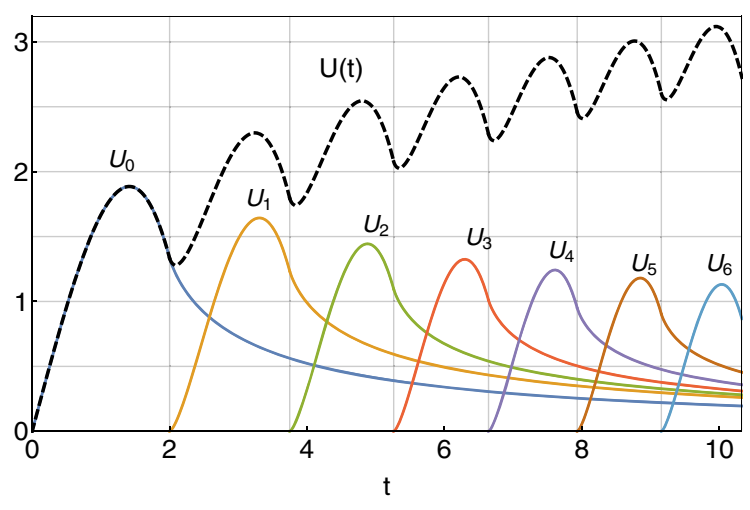

(b) $U_{k}(t)$

FIG. 7. (a) Kinetic and gradient energies of partial solutions. (b) Potential energies of these solutions. 
The vertical grid lines correspond with instants of time $t_{k}=$ $2 \sqrt{a_{k}}$ at which new partial solutions appear. The abrupt decreasing of the energy $K_{k}(t)$ which manifests in the presence of spikes is caused by the appearance of the new partial solution $\phi_{k+1}$ and, consequently, by a quick decrease of the support size of $\phi_{k}$. We have plotted also the first four straight lines (3.10) and (3.12) for $k=1,2,3$. They are marked by dashed lines. The picture in Fig. 7(a) shows that the dominating contribution to the kinetic and gradient energy is associated with the partial solution $k=0$. Its energy grows as $a_{0} t$ for large $t .{ }^{4}$ Note that the partial solution $k=0$ is in contact with the discontinuity at $x= \pm t$. This discontinuity can be seen as a reservoir of infinite energy. The energy is transferred first into $\phi_{0}(t, x)$ and then it is transferred to the other partial solutions $\phi_{k}(t, x)$. The central partial solutions carry less energy than partial solutions localized close to the light cone.

Now we shall look at the potential energy of the partial solutions. The potential energy associated with the partial solution $\phi_{0}(t, x)$ reads

$U_{0}(t)= \begin{cases}2 a_{0} t-\frac{t^{3}}{3} & \text { for } 0 \leq t \leq t_{0} \\ 2 a_{0} t-\frac{t^{3}}{3}+\frac{1}{3}\left(t^{2}-4 a_{0}\right)^{3 / 2} & \text { for } t \geq t_{0}\end{cases}$

which has the following asymptotic behavior for large $t$ :

$$
U_{0}(t) \approx \frac{2 a_{0}^{2}}{t}+\mathcal{O}\left(t^{-3}\right)
$$

Similarly we find the potential energy of partial solutions with $k=1,2, \ldots$ They are given by

$$
U_{k}(t)= \begin{cases}U_{k}^{(-)}(t)=R_{k}(t) & \text { for } \quad t_{k-1} \leq t \leq t_{k} \\ U_{k}^{(+)}(t)=R_{k}(t)+S_{k}(t) & \text { for } \quad t \geq t_{k}\end{cases}
$$

where

$$
\begin{aligned}
R_{k}(t) & =\left[\frac{4}{3} a_{k}-4 b_{k}-\frac{t^{2}}{3}\right] c_{k-1}(t)+4 b_{k} t \operatorname{ArcTanh}\left(\frac{c_{k-1}(t)}{t}\right), \\
S_{k}(t) & =-\left[\frac{4}{3} a_{k-1}-4 b_{k}-\frac{t^{2}}{3}\right] c_{k}(t)-4 b_{k} t \operatorname{ArcTanh}\left(\frac{c_{k}(t)}{t}\right) .
\end{aligned}
$$

We have eliminated logarithmic terms in $R_{k}$ and $S_{k}$ using the second one of relations (2.3). The last formula implies that, for large $t$, the expression for $U_{k}(t)$ behaves as

\footnotetext{
${ }^{4}$ This is exactly half of the total energy of the wave; see (3.22).
}

$U_{k}(t) \approx 2\left[\left(a_{k}-a_{k-1}\right)\left(a_{k}+a_{k-1}-2 b_{k}\right)\right] \frac{1}{t}+\mathcal{O}\left(t^{-2}\right)$.

It shows the potential energy of each partial solution decreasing as $t^{-1}$ for large $t$.

The energy of a single partial solution is given by a sum of expressions (3.9) and (3.13)

$E_{0}(t)=\left\{\begin{array}{ll}E_{0}^{(-)}(t)=X_{0}(t) & \text { for } \quad 0 \leq t \leq t_{0} \\ E_{0}^{(+)}(t)=X_{0}(t)+Y_{0}(t) & \text { for } \quad t \geq t_{0}\end{array}\right.$,

where

$$
X_{0}(t):=2 a_{0} t, \quad Y_{0}(t):=-a_{0} c_{0}(t) .
$$

Similarly, for $k=1,2, \ldots$ the energy of partial solutions is obtained adding (3.11) and (3.15). It reads

$E_{k}(t)= \begin{cases}E_{k}^{(-)}(t)=X_{k}(t) & \text { for } \quad t_{k-1} \leq t \leq t_{k} \\ E_{k}^{(+)}(t)=X_{k}(t)+Y_{k}(t) & \text { for } t \geq t_{k}\end{cases}$

where

$X_{k}(t):=P_{k}(t)+R_{k}(t)=\left[\frac{b_{k}^{2}}{a_{k-1}}-2 b_{k}+a_{k-1}\right] c_{k-1}(t)$,

$Y_{k}(t):=Q_{k}(t)+S_{k}(t)=-\left[\frac{b_{k}^{2}}{a_{k}}-2 b_{k}+a_{k}\right] c_{k}(t)$.

In Fig. 8 we plot the energies (3.17) and (3.19) of partial solutions together with individual contributions from kinetic gradient and potential part. It is quite notable that the total energy of the wave grows exactly linearly with time. In order to show it we consider a total energy of the wave at $t$ belonging to the interval $t_{k-1} \leq t \leq t_{k}$. Since the wave consists on first $k+1$ partial solutions then it has the energy

$$
E(t)=\sum_{i=0}^{k} E_{i}(t)=\sum_{i=0}^{k-1} E_{i}^{(+)}(t)+E_{k}^{(-)}(t),
$$

where $E_{i}^{(+)}(t)=X_{i}(t)+Y_{i}(t)$ and $E_{k}^{(-)}(t)=X_{k}(t)$. Expression (3.22) can be cast in the form

$$
E(t)=X_{0}(t)+\sum_{i=1}^{k}\left[Y_{i-1}(t)+X_{i}(t)\right]
$$




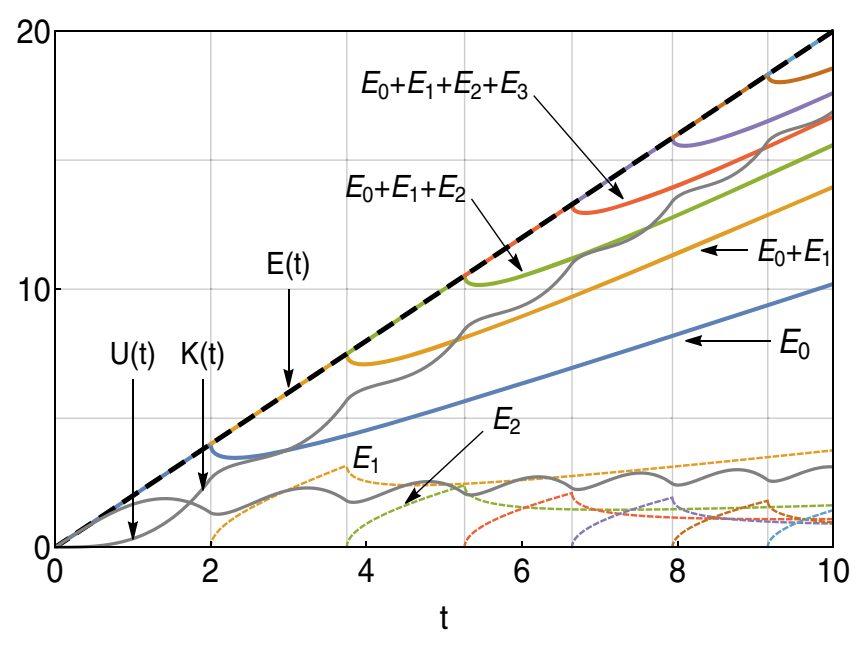

FIG. 8. Total energies of partial solutions $E_{k}(t)$ and total energy $E(t)=K(t)+U(t)$ of the wave. The dashed line represents total energy of the wave.

Making use of the relation $b_{1}=2 a_{0}$ and the first one of relations (2.3) we get

$$
\begin{aligned}
X_{1}(t)+Y_{0}(t) & =\left[\frac{b_{1}^{2}}{a_{0}}-2 b_{1}\right] c_{0}(t)=0, \\
X_{i}(t)+Y_{i-1}(t) & =\left[\frac{b_{i}^{2}-b_{i-1}^{2}}{a_{i-1}}-2 b_{i-1}-2 b_{i}\right] c_{i-1}(t)=0, \\
i & =1,2, \ldots k .
\end{aligned}
$$

It shows that the energy of the wave inside the light cone is given by the expression $X_{0}(t)$

$$
E(t)=2 a_{0} t
$$

This result shows that the energy of the wave inside the light cone grows linearly with time.

\section{SOLUTIONS}

In this section we go back to regular initial data with the deltalike profile of the time derivative of the field. First we make some important remarks on the numerical solutions we have adopted. Then we present numerical solutions of shocklike waves and give the analytical solution for the initial phase of the formation of the wave. Finally, we compare some numerical simulations containing the formation of shocklike waves with the results of the scattering of compact oscillons.

\section{A. Remarks on the numerical method and its reliability}

We have made use of the $4^{\text {th }}$ order Runge-Kutta method with a regularly discretized space dimension and have advanced the initial conditions with constant time steps. We have checked that the numerical solutions usually start to converge for the space resolution of at least about $5 k$ sites.
The very small features of the field (small oscillon like structures) do not ever converge to a certain solution even for very large resolutions (about $1 M$ sites). The sensibility that these structures have on initial conditions (which are slightly affected by small changes in simulation resolution) has been the subject of thorough discussion [18]. This means that the very small structures observed in these simulations (i.e., oscillons of width < 0.1 ) are not a $100 \%$ accurate description of the field, although they are qualitatively accurate. Also, our simulations have been implemented twice, and independently, and the results converge.

\section{B. Numerical results for triangular and Gaussian initial data}

Here we shall present the results of the numerical evolution of the initial data

$$
\left.\phi(t, x)\right|_{t=0}=0,\left.\quad \partial_{t} \phi(t, x)\right|_{t=0}=a \delta_{\epsilon}(x),
$$

where $\delta_{\epsilon}(x)$ is given by both the Gaussian function (2.12) and the triangular function (2.13). We study how the numerical solution changes in dependence with the value of the parameter $\epsilon$. The results are presented on spacetime diagrams where the value of the field is marked by the gradient color. We also plot the energy density of the system

$$
\mathcal{H}=\frac{1}{2}\left(\partial_{t} \phi\right)^{2}+\frac{1}{2}\left(\partial_{x} \phi\right)^{2}+|\phi| .
$$

In Fig. 9 we show the evolution of the signum-Gordon field for the deltalike initial profile of $\partial_{t} \phi$. We consider $\epsilon=\left\{10^{-6}, 2 \times 10^{-5}, 4 \times 10^{-5}, 5 \times 10^{-5}\right\}$. For $\epsilon=10^{-6}$ the numerical solution looks very similar to the exact shock wave. After increasing $\epsilon$ by a factor 20 we see that the wave breaks down for $t>4$. Looking at the energy density in Fig. 9(d) we can see an initial phase of the formation of jets. This behavior is even more visible for $\epsilon=4 \times 10^{-5}$ and $\epsilon=5 \times 10^{-5}$. Looking in more detail at these jets we see that they contain structures which are very similar to oscillons. Some of these oscillons interact with others which leads to quite complex structures. The region of existence of a shocklike wave configuration of the signum-Gordon field shrinks when $\epsilon$ decreases.

We have also looked at higher values of $\epsilon$. For $\epsilon=2 \times 10^{-4}$, see Fig. 10(a,b), the region of spacetime being the support of the wave has a diamond-shaped form. At the left and right edge of the diamond two oscillons emerge. They move in opposite directions with relatively high speeds. In the central part of the diagram we see the formation of a certain number of slow oscillons. They interact with each other by scattering and by emission and absorption of radiation.

Our choice of a triangular shape for the $\delta_{\epsilon}$ function allows us to obtain an exact oscillon. In the case of the 


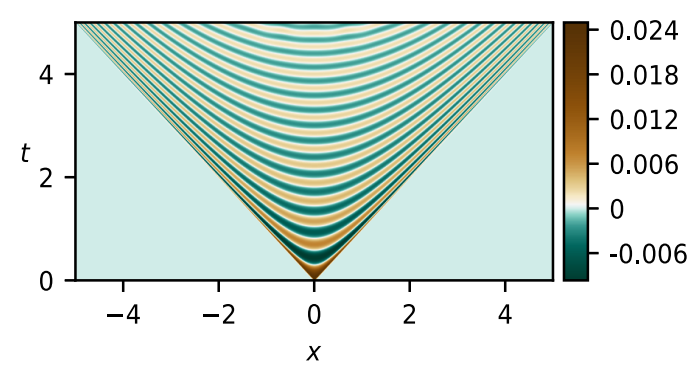

(a) $\epsilon=10^{-6}$

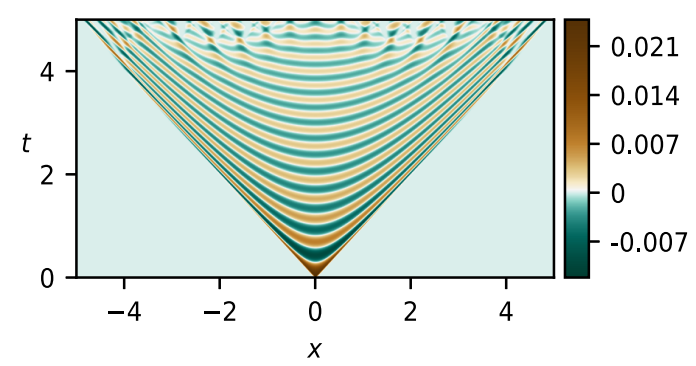

(c) $\epsilon=2 \cdot 10^{-5}$

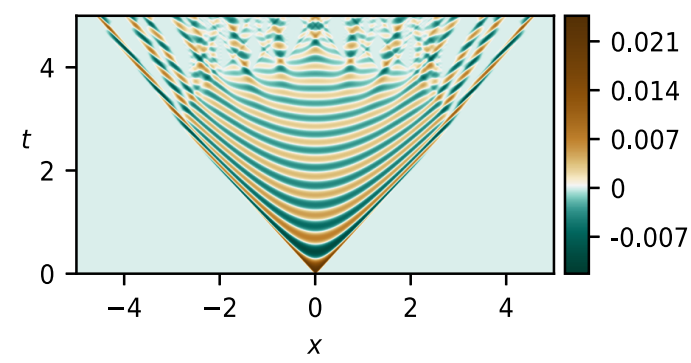

(e) $\epsilon=4 \cdot 10^{-5}$

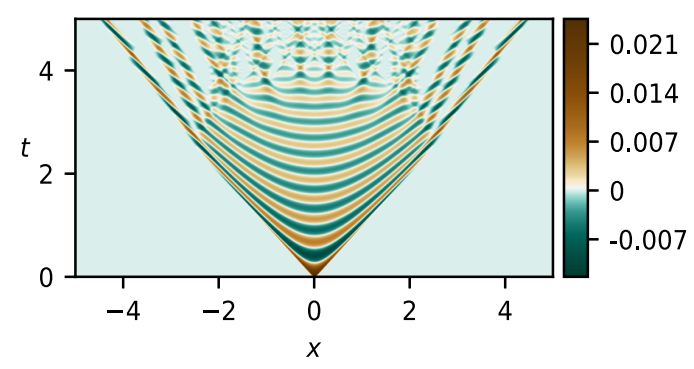

(g) $\epsilon=5 \cdot 10^{-5}$

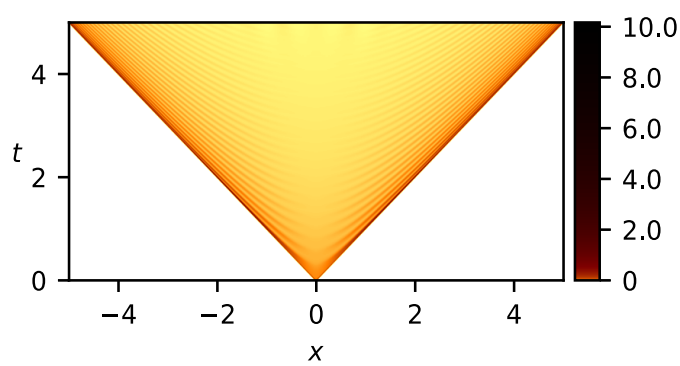

(b) $\epsilon=10^{-6}$

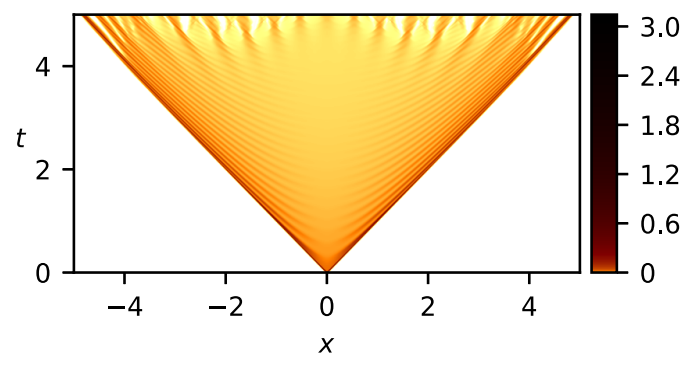

(d) $\epsilon=2 \cdot 10^{-5}$

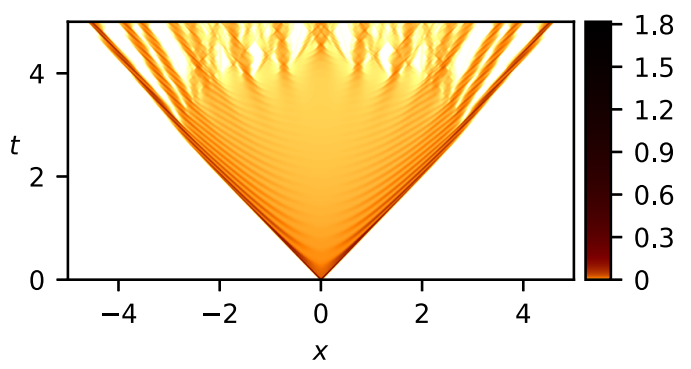

(f) $\epsilon=4 \cdot 10^{-5}$

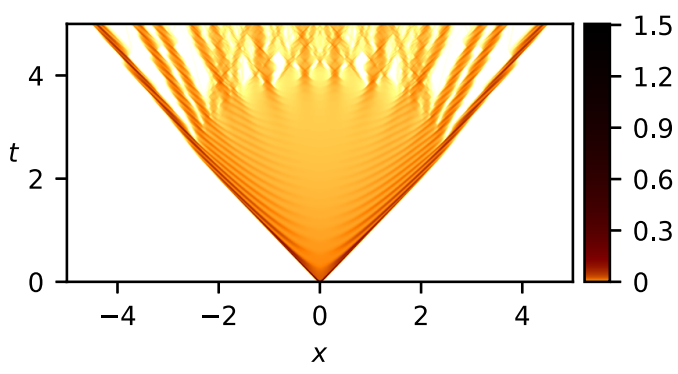

(h) $\epsilon=5 \cdot 10^{-5}$

FIG. 9. The case of triangular form of $\delta_{\epsilon}$. The decay of the shock wave in function of $\epsilon$ for $a=0.05$. Field $\phi(t, x)$ (left) and its energy density (right).

simplest exact oscillon the initial profile of velocity is an isosceles triangle with sides that form the angle $\alpha=\pi / 4$ with the $x$-axis. Hence, the ratio of its height $a \delta_{\epsilon}(0)=\frac{a}{2 \sqrt{\pi \epsilon}}$ by a half of size of its base $2 \sqrt{\pi \epsilon}$ must be equal to unity, i.e., $\frac{a}{4 \pi \epsilon}=1$. It means that $\epsilon=\frac{a}{4 \pi}$ is a special value that gives the simplest exact oscillon with the support size $2 \sqrt{a}$. Taking $a=0.05$ one gets $\epsilon=\frac{1}{80 \pi} \approx 0.0039788 \approx 4 \times 10^{-3}$.
The numerical solution corresponding with this case is presented in Fig. 11.

We have also checked the evolution of the signum-Gordon field for higher values of $\epsilon$. A solution obtained for $\epsilon=$ $4 \times 10^{-2}$ is sketched in Fig. 12. The numerical solution represents the collision of two segments of self-dual solutions which results in the appearance of radiation that consists on many oscillons. 


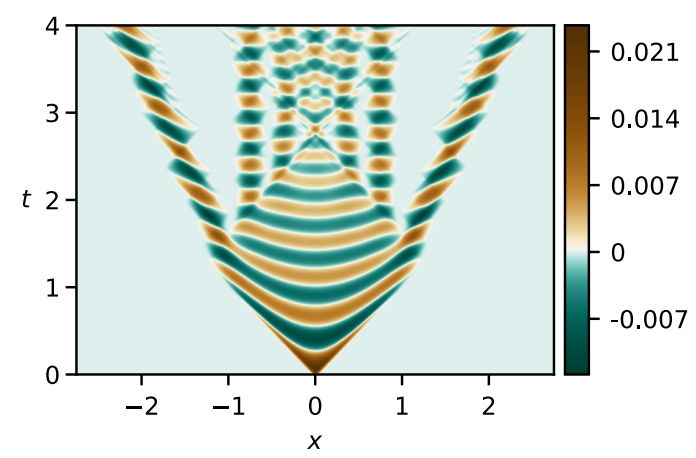

(a) $\phi(t, x)$

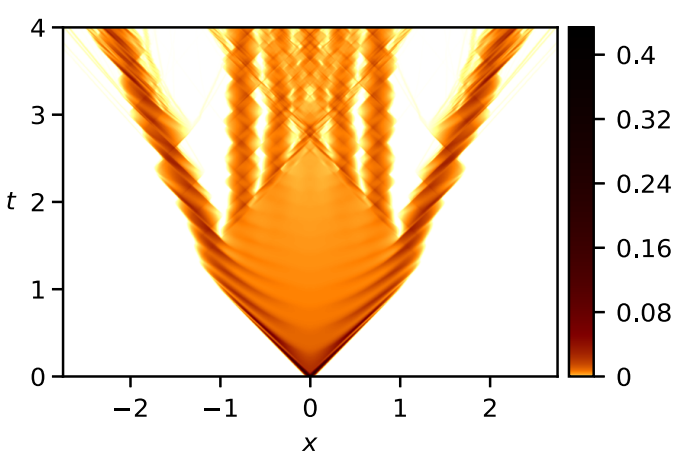

(b) $\mathcal{H}(t, x)$

FIG. 10. The case of triangular form of $\delta_{\epsilon}: a=0.05$ and $\epsilon=2 \times 10^{-4}$. (a) Field $\phi$ and (b) its energy density.

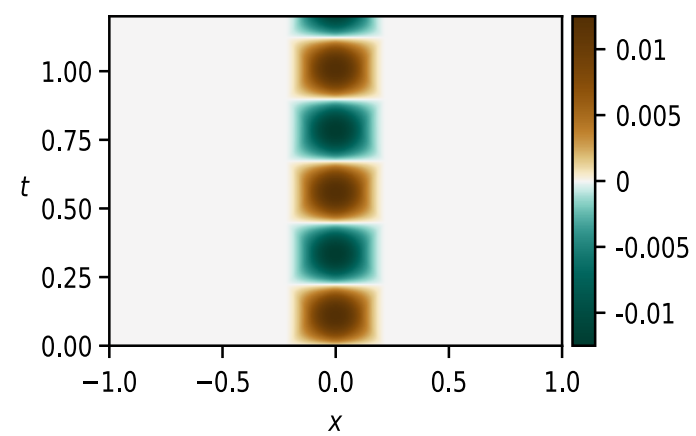

(a) $\phi(t, x)$

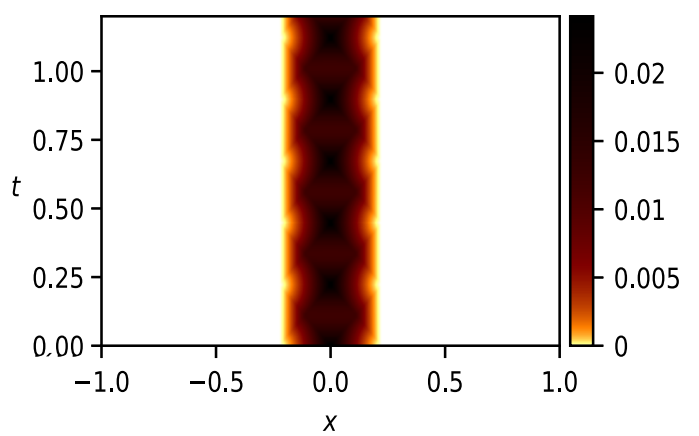

(b) $\mathcal{H}(t, x)$

FIG. 11. The case of triangular form of $\delta_{\epsilon}: a=0.05$ and $\epsilon=0.0039788 \approx 4 \times 10^{-3}$. In this case the numerical solution corresponds with the exact oscillon.

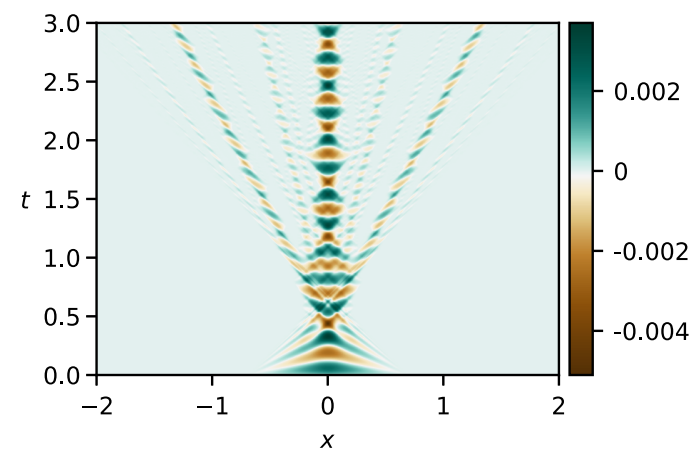

(a) $\phi(t, x)$

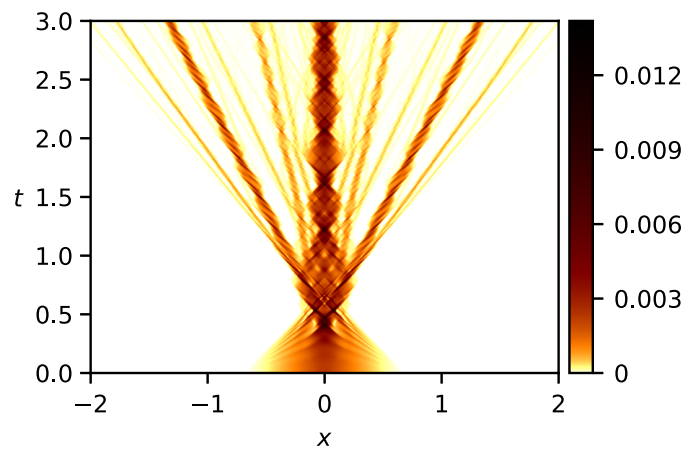

(b) $\mathcal{H}(t, x)$

FIG. 12. The case of triangular form of $\delta_{\epsilon}: a=0.05$ and $\epsilon=4 \times 10^{-2}$.

In order to check to which extent our results depend on the choice of the form of $\delta_{\epsilon}(x)$, we also performed simulations taking its Gaussian version. The results are shown in Figs. 13-15. Comparing these figures with the previous ones we do not see much difference between the triangular and the Gaussian cases. Figures 10 and 13 are very similar. More differences appear when comparing the Gaussian case for $\epsilon=\frac{1}{80 \pi}$ with the triangular case suitable for exact oscillon. Contrary to Fig. 11, the solution shown in Fig. 14 has a border which is not a straight line. This is pretty clear from the picture of the energy density shown in Fig. 15(b). A counterpart of the exact oscillon obtained in this case is a solution whose support shrinks and expands periodically. Similarly in the case $\epsilon=4 \times 10^{-2}$ a region with a high value of the energy density shrinks and expands with certain regularity; see Fig. 15. The maximum size of the regions with a high value of the energy density decreases with time because of the emission of oscillons. 


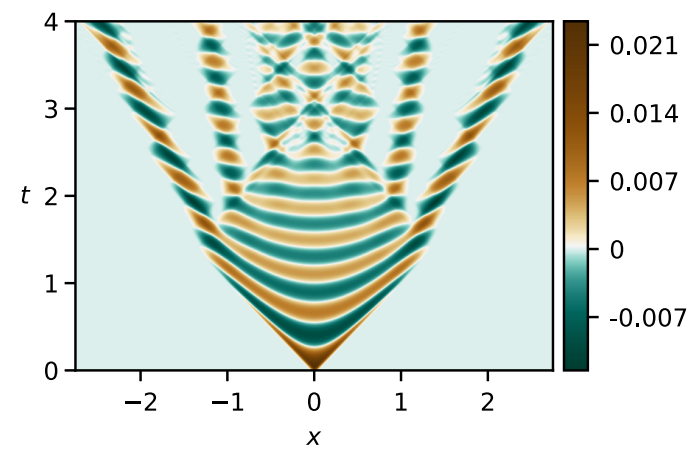

(a) $\phi(t, x)$

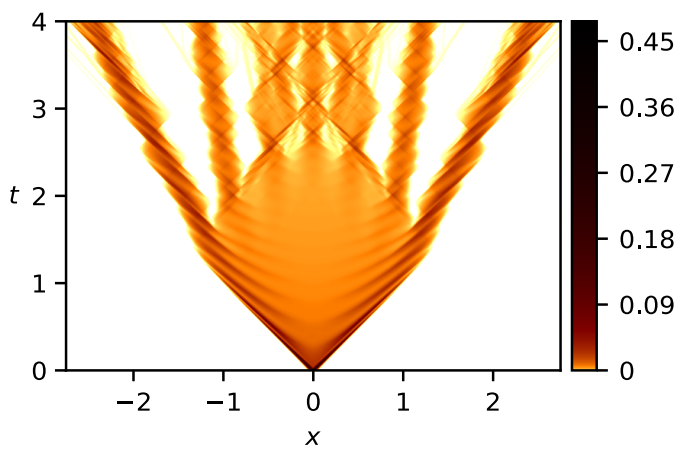

(b) $\mathcal{H}(t, x)$

FIG. 13. The case of Gaussian form of $\delta_{\epsilon}: a=0.05$ and $\epsilon=2 \times 10^{-4}$. (a) Field $\phi$ and (b) its energy density.

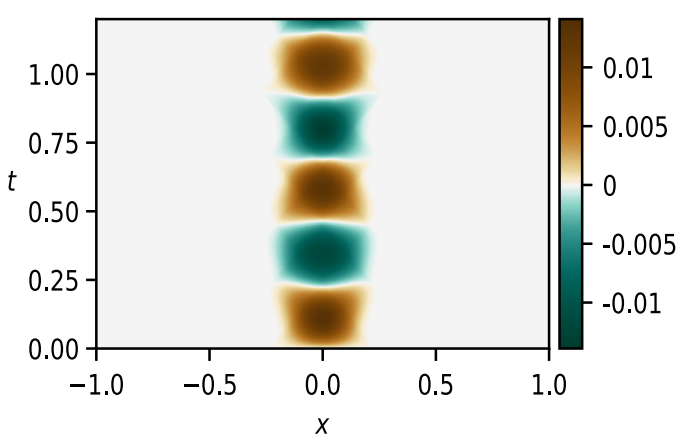

(a) $\phi(t, x)$

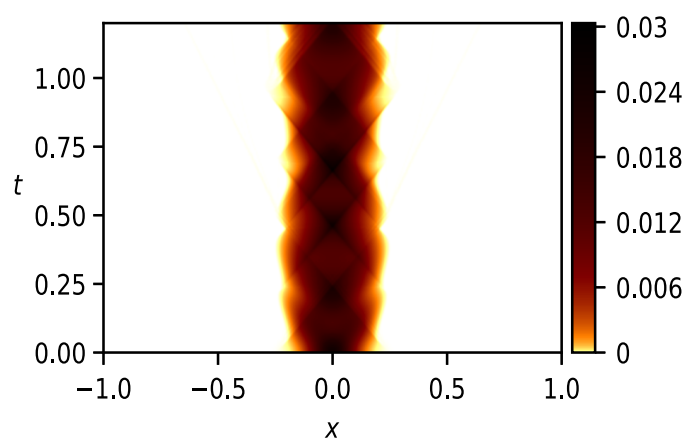

(b) $\mathcal{H}(t, x)$

FIG. 14. The case of Gaussian form of $\delta_{\epsilon}: a=0.05$ and $\epsilon=4 \times 10^{-3}$.

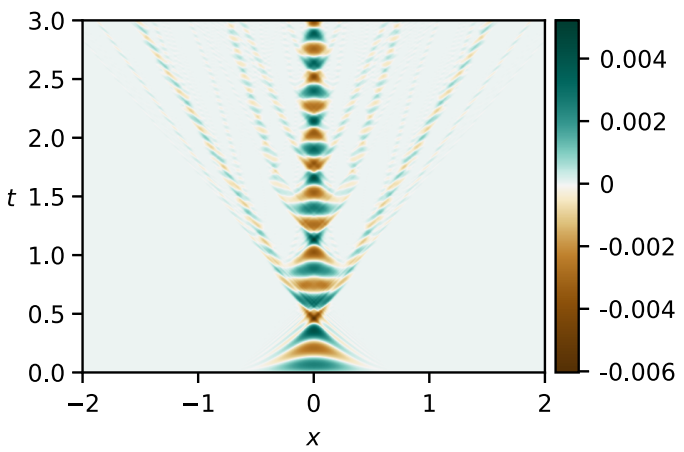

(a) $\phi(t, x)$

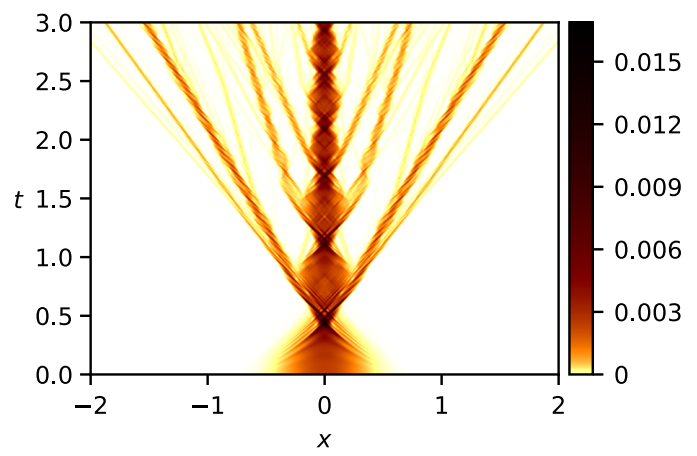

(b) $\mathcal{H}(t, x)$

FIG. 15. The case of Gaussian form of $\delta_{\epsilon}: a=0.05$ and $\epsilon=4 \times 10^{-2}$.

\section{Exact solution in an initial stage of evolution}

In this section we shall study some analytical expressions that describe a shocklike wave solution. Having in mind the results of our numerical simulations we do not expect to obtain the exact solution for arbitrarily long times. On the other hand, the initial triangular data are simple enough to get some analytical results describing a wave in its initial stage of evolution. It would be interesting to compare such solutions with their counterparts that form an exact shock wave.
The triangular initial data (4.1) with $\epsilon \equiv \frac{\varepsilon^{2}}{4 \pi}$ is given on a segment $x \in[-\varepsilon, \varepsilon]$, where the triangular shape is given by the expression

$\delta_{\varepsilon}(x)=\frac{1}{\varepsilon^{2}}[(\varepsilon+x) \theta(\varepsilon+x) \theta(-x)+(\varepsilon-x) \theta(\varepsilon-x) \theta(x)]$.

The energy of such initial field configuration equals to (2.21) or $E=\frac{a^{2}}{3 \varepsilon^{2}}$. This energy is finite and conserved during the evolution. The initial data are symmetric under spatial 


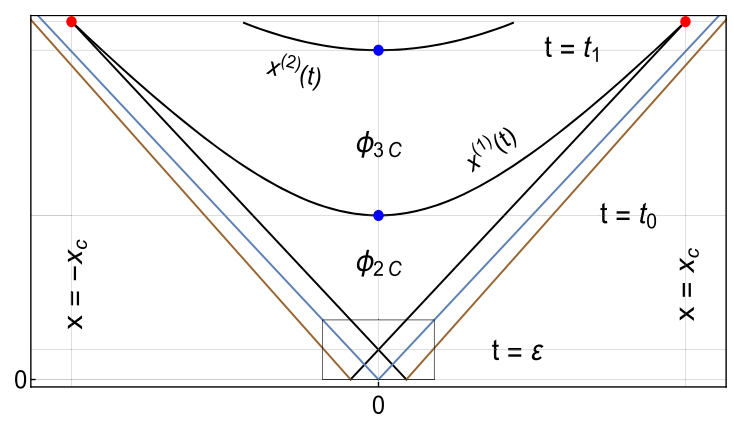

(a)

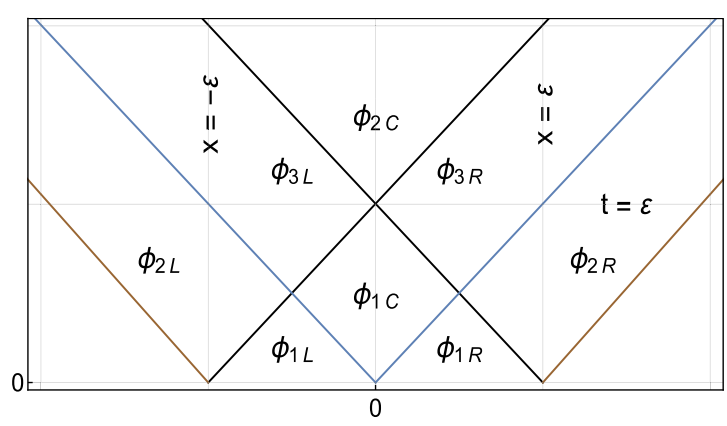

(b)

FIG. 16. Partial solutions for triangular initial data: $a=0.05$ and $\epsilon=2 \times 10^{-4}(\varepsilon=0.05013)$.

reflection and so is the solution. This solution consists on partial solutions that have the general form given by expression (1.2). It is known that initial data for the signum-Gordon field determine the number and the size of the supports of the partial solutions. The restriction to the triangular initial data still leaves a freedom of a choice of the parameters $a$ and $\varepsilon$. These parameters determine the inclination of the sides of the triangle. According to Ref. [21] an inclination of the velocity profile determines whether the support of the solution spreads out, remains unchanged (the exact oscillon), or shrinks. Since we are interested in solutions which are similar to exact shock waves, then we will study a triangular shape with the height $a \delta_{\varepsilon}(0)=\frac{a}{\varepsilon}>\varepsilon$. An example of such a solution with $a=$ 0.05 and $\epsilon=2 \times 10^{-4}$ (thus $\frac{a}{\varepsilon^{2}}=\frac{a}{4 \pi \epsilon} \approx 19.89>1$ ) is shown in Fig. 10. In Fig. 16 we present domains of various partial solutions that compose the solution in its initial stage of evolution. Figure 16(b) shows a blowup of the rectangular region at Fig. 16(a). The partial solution $\phi_{1 L}(t, x)$ can be obtained directly from the initial data and it reads

$$
\phi_{1 L}(t, x)=\frac{a}{\varepsilon^{2}}[x+\varepsilon] t-\frac{t^{2}}{2} .
$$

Its symmetric counterpart is given by $\phi_{1 R}(t, x)=\phi_{1 L}(t,-x)$. These two solutions are restricted to the interior of the past light cones of the two events $(t, x)=\left(\frac{\varepsilon}{2}, \pm \frac{\varepsilon}{2}\right)$. Note that (4.3) and (2.15) differ only by the expression $-\frac{t^{2}}{2}$.

The partial solution $\phi_{2 L}(t, x)$ interpolates between $\phi_{1 L}(t, x)$ and the vacuum solution $\phi=0$. It matches $\phi_{1 L}(t, x)$ at the light cone $x=-\varepsilon+t$ and the vacuum at $x=-\varepsilon+v t$, where $v<0$. This solution reads

$$
\phi_{2 L}(t, x)=\frac{(x+\varepsilon-v t)^{2}}{2\left(1-v^{2}\right)}, \quad v=-1+\frac{\varepsilon^{2}}{a} .
$$

Its symmetric counterpart is given by $\phi_{2 R}(t, x)=\phi_{2 L}(t,-x)$. Note that the subluminal zero $x=-\varepsilon+v t$ crosses the light cone $x=-t$ at $t_{c}=\frac{a}{\varepsilon}$. It results in the compactness of a region of spacetime in which $\phi_{2 L}$ holds. Note also that $\phi_{1 L}$ and $\phi_{2 L}$ (as well as $\phi_{1 R}$ and $\phi_{2 R}$ ) are given by expressions which are identical with the formulas that describe the self-similar solutions discussed in [21]. The basic difference is that in the present case the supports of the self-similar partial solutions are restricted to a region corresponding with the exterior of the future light cone of the event $(t, x)=(0,0)$. The region inside this light cone contains some new partial solutions.

In order to obtain the partial solution $\phi_{1 C}(t, x)$, we assume its positivity (i.e., we choose a term " $-\frac{t^{2}}{2}$,") and impose the following matching conditions $\phi_{1 C}(t,-t)=$ $\phi_{1 L}(t,-t)$ and $\phi_{1 C}(t, t)=\phi_{1 R}(t, t)$. It gives

$$
\phi_{1 C}(t, x)=\frac{1}{1+v}\left[\varepsilon t-\frac{x^{2}+t^{2}}{2}\right]-\frac{t^{2}}{2} .
$$

This solution remains valid in a compact region delimited by the intersection of the future light cone of the event $(0,0)$ and the past light cone of the event $(\varepsilon, 0)$. The events $\left(\frac{\varepsilon}{2}, \pm \frac{\varepsilon}{2}\right)$ correspond with two points at the Minkowski diagram at which new partial solutions $\phi_{3 L}$ and $\phi_{3 R}$ emerge. The solution $\phi_{3 L}(t, x)$ satisfies the matching conditions $\phi_{3 L}(t,-t)=\phi_{2 L}(t,-t)$ and $\phi_{3 L}(t,-\varepsilon+t)=$ $\phi_{1 C}(t,-\varepsilon+t)$. It takes the form

$$
\begin{aligned}
\phi_{3 L}(t, x)= & \frac{1}{1+v}\left[\frac{\varepsilon}{2}(x+t)-\frac{1}{4}(x+t)^{2}\right]+\frac{(x-t)^{2}}{8}-\frac{t^{2}}{2} \\
& +\frac{1}{2\left(1-v^{2}\right)}\left[\varepsilon+(1+v) \frac{x-t}{2}\right]^{2}
\end{aligned}
$$

Figure 16 shows that, in the limit $\varepsilon \rightarrow 0$, all the supports of the partial solutions presented above shrink to points. Moreover, in this limit the gradients of the partial solutions become singular. It reflects the emergence of discontinuities of the field at the light cone. As a result, there are no counterparts for these solutions in the set of partial solutions describing the exact shock wave.

On the other hand, the partial solution $\phi_{2 C}(t, x)$ is a counterpart of the first partial solution $\phi_{0}(t, x)=\frac{1}{4}\left(x^{2}-\right.$ $\left.t^{2}\right)+a_{0}$ that composes the exact shock wave. This solution matches $\phi_{3 L}(t, x)$ at $x=\varepsilon-t$ and $\phi_{3 R}(t, x)$ at $x=-\varepsilon+t$. It has the form 
$\phi_{2 C}(t, x)=\frac{x^{2}-b(t)}{2(1-v)}$, where $b(t):=-v t^{2}+2 \varepsilon t-2 a$.

Indeed, in the limit $\varepsilon \rightarrow 0,(v \rightarrow-1)$ the solution $\phi_{2 C}(t, x)$ tends to $\phi_{0}(t, x)$ with $a_{0}=\frac{1}{2} a$ [see Fig. 1(a) for the meaning of $\left.a_{0}\right]$. The coefficient $b(t)$ satisfies $b(t) \geq 0$ for $t \leq t_{0}$ where

$$
t_{0}=-\frac{\varepsilon}{v}\left[\sqrt{\frac{1-v}{1+v}}-1\right] .
$$

Thus, $\phi_{2 C}\left(t_{0}, 0\right)=0$. Note that $t_{0} \rightarrow \sqrt{2 a}=2 \sqrt{a_{0}}$ in the limit $\varepsilon \rightarrow 0$. For $t>t_{0}$ the zero of $\phi_{2 C}(t, x)$ splits into two zeros $x^{(1)}(t)= \pm \sqrt{b(t)}$ that move in opposite directions. Trajectories of the zeros $x^{(1)}(t)$ delimit the domain of the solution $\phi_{2 C}(t, x)$. In the case of exact shock wave $(\varepsilon=0)$ this region is restricted from below by the light cone $x=$ $\pm t$ and from above by a hyperbola $x(t)= \pm \sqrt{t^{2}-2 a}$. This situation changes qualitatively for solution (4.7), which, contrary to its counterpart, is delimited from below by the future light cone $x= \pm \varepsilon \mp t$ of the event $(t, x)=(\varepsilon, 0)$ and from above by a hyperbolalike curve $x^{(1)}(t)$. The intersection of the line $x=-\varepsilon+t$ with the curve $x^{(1)}(t)$ determines an instant of time

$$
t_{c}=\frac{a}{\varepsilon}(2-\sqrt{1-v}),
$$

at which both supports of the solution $\phi_{2 C}(t, x)$ shrink to zero. It means that $\phi_{2 C}(t, x)$ is restricted to a compact region on the Minkowski diagram. This region is delimited by the endpoints $\pm x_{c}$ where $x_{c}:=-\varepsilon+t_{c}$. The events $\left(t_{c}, \pm x_{c}\right)$ are marked in Fig. 16(a). Note that $t_{c} \rightarrow \infty$ for $\varepsilon \rightarrow 0$.

The partial solution that matches $\phi_{2 C}(t, x)$ at $x^{(1)}(t)=$ $\pm \sqrt{b(t)}$ is symmetric in the variable $x$ and negative valued. Imposing matching conditions at $x=\sqrt{b(t)}$ on the central partial solution $\phi_{3 C}(t, x)=F(x+t)+G(x-t)+$ $\frac{t^{2}}{2}$ we get

$$
\begin{aligned}
F(\sqrt{b(t)}+t)+G(\sqrt{b(t)}-t)+\frac{t^{2}}{2} & =0, \\
F^{\prime}(\sqrt{b(t)}+t)+G^{\prime}(\sqrt{b(t)}-t) & =\frac{a \sqrt{b(t)}}{2(1-v)},
\end{aligned}
$$

where $F^{\prime}(s)=\frac{d F(s)}{d s}$ and similarly for $G^{\prime}(s)$. Solving these equations we get

$$
\begin{aligned}
\phi_{3 C}(t, x)= & \frac{1}{1+v}\left[H(t+x)+H(t-x)-2 H\left(t_{0}\right)\right] \\
& +\frac{1}{2}\left(t^{2}-t_{0}^{2}\right)+\alpha^{(-)}\left(t^{2}+x^{2}-t_{0}^{2}\right) \\
& -2 \varepsilon \alpha^{(+)}\left(t-t_{0}\right),
\end{aligned}
$$

where the coefficients $\alpha^{( \pm)}$have the form $\alpha^{( \pm)}=\frac{1}{2}\left(\frac{1}{1-v} \pm\right.$ $\left.\frac{2}{1+v}\right)$. The function $H(z)$ stands for the integral $H(z):=$ $\int d z \sqrt{c(z)}$, where $c(z)=-v z^{2}+2 \varepsilon z-\varepsilon^{2}$, and it reads

$$
\begin{aligned}
H(z)= & \frac{1}{2}\left(z-\frac{\varepsilon}{v}\right) \sqrt{c(z)} \\
& -\frac{1}{2}\left(\varepsilon^{2} \frac{1-v}{(-v)^{\frac{3}{2}}} \ln \left[2 a\left(\frac{\varepsilon}{\sqrt{-v}}+z \sqrt{-v}+\sqrt{c(z)}\right)\right]\right) .
\end{aligned}
$$

The integration constant is fixed by the condition $\phi_{3 C}\left(t_{0}, 0\right)=0$. The partial solution (4.10) is negative valued on the segment $-\sqrt{b(t)}<x<\sqrt{b(t)}$ in the interval of time $t_{0}<t<t_{1}$. At $t=t_{1}$ another zero arises, namely $\phi_{3 C}\left(t_{1}, 0\right)=0$. Due to the complexity of the expression (4.10) we cannot give an exact solution to this equation. Solving numerically this equation for $a=0.05$ and $\varepsilon=$ 0.05013 we get $t_{1} \approx 0.554$.

The validity of our numerical solution is determined by the instant of time $t_{c}$, given by (4.9) and in its central region by a hyperbolalike curve $x^{(2)}(t)$, which describes the second zero of $\phi_{3 C}(t, x)$, i.e., $\phi_{3 C}\left(t, x^{(2)}(t)\right)=0$. In our example, $t_{c} \approx 0.602$. In order to get a solution valid for $t>t_{c}$ one has to construct a solution inside the future light cones of the events $\left(t_{c}, \pm x_{c}\right)$ where $x_{c}=-\varepsilon+t_{c}$. Such a solution should match $\phi_{3 C}(t, x)$ at $x= \pm\left[x_{c}-\left(t-t_{c}\right)\right]$ and $\phi_{3 R / L}(t, x)$ at $x= \pm\left[x_{c}+\left(t-t_{c}\right)\right]$. Since $\phi_{3 C}<0$ in the vicinity of the matching point and $\phi_{3 R / L}>0$ then the partial solution inside the future light cone of the event $\left(t_{c}, \pm x_{c}\right)$ should consist of at least two partial solutions with opposite signs. Unfortunately, the complexity of the expression (4.10) makes it impossible to obtain an exact expression for such solutions. For the same reason we cannot give an exact expression for another partial solution in the central region above the hyperbola $x^{(2)}(t)$.

In Fig. 17 we show the exact shocklike waves obtained for $a=0.05$ and $\epsilon=2 \times 10^{-4}$ in three instants of time

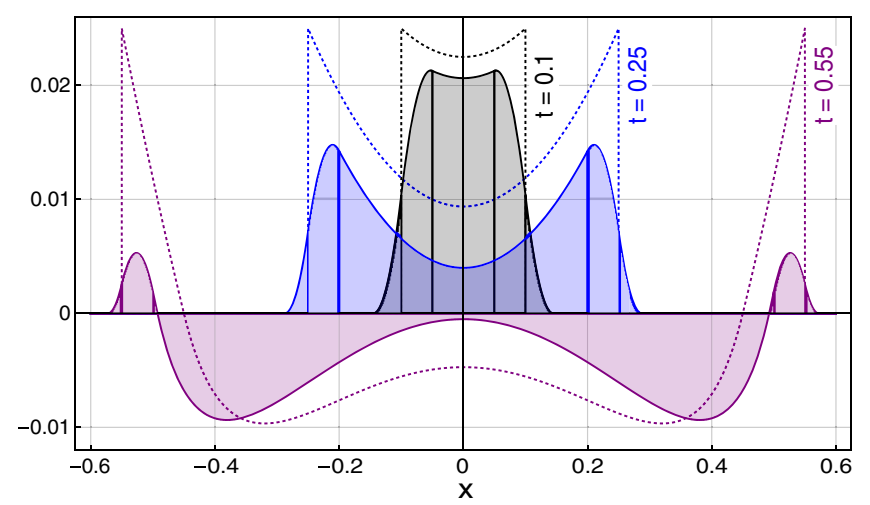

FIG. 17. Evolution of a shocklike wave characterized by $a=$ 0.05 and $\epsilon=2 \times 10^{-4}$, and evolution of the exact shock wave for $t=0.1, t=0.25$, and $t=0.55$. 


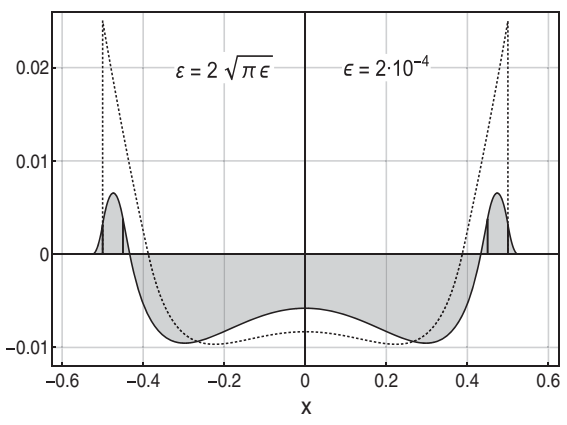

(a)

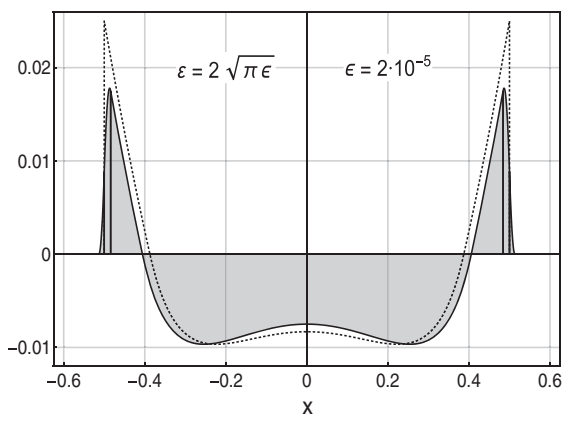

(b)

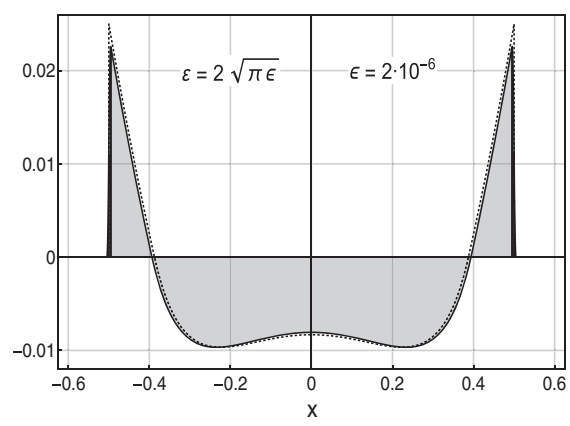

(c)

FIG. 18. The shocklike wave at $t=0.5$ for $a=0.05$ and (a) $\epsilon=2 \times 10^{-4}$, (b) $\epsilon=2 \times 10^{-5}$, and (c) $\epsilon=2 \times 10^{-6}$. For $\epsilon \rightarrow 0$ the shock-wave-like solution (solid curve) tends to the exact shock wave solution (dotted curve).

$t=0.1, t=0.25$ and $t=0.55$. The dotted line represents the signum-Gordon field corresponding with an exact shock wave configuration with $a_{0}=\frac{1}{2} a$. Two external bumps localized in the vicinity of the future light cone of the event $(t, x)=(0,0)$ decrease with time.

Figure 18 shows the exact shock wave and shocklike wave solutions at $t=0.5$. The regular (without discontinuities) solutions are taken for three different values of the parameter $\varepsilon=2 \sqrt{\pi \epsilon}$ given by $\epsilon=2 \times 10^{-4}, \epsilon=2 \times 10^{-5}$, and $\epsilon=2 \times 10^{-6}$. The shocklike wave solution tends to the exact shock wave in the limit $\epsilon \rightarrow 0$.

Knowing the exact partial solutions, we can write explicit expressions for the energy density carried by each piece of the solution:

$$
\begin{gathered}
u_{1 C}(t, x)=\frac{(2+v) t^{2}-2 \varepsilon t-v x^{2}+\varepsilon^{2}}{2(1+v)^{2}} \\
u_{2 C}(t, x)=\frac{v t^{2}-2 t \varepsilon+(2-v) x^{2}}{2(1-v)^{2}}+\frac{(3-v) \varepsilon^{2}}{2(1-v)^{2}(v+1)} \\
u_{1 L}(t, x)=\frac{t^{2}+(x+\varepsilon)^{2}}{2(1+v)^{2}} \\
u_{2 L}(t, x)=\frac{(x+\varepsilon-v t)^{2}}{\left(1-v^{2}\right)^{2}}
\end{gathered}
$$

$$
\begin{aligned}
u_{3 L}(t, x)= & \frac{1}{2\left(1-v^{2}\right)^{2}}\left[-v\left(1-v^{2}\right)\left(t^{2}-x^{2}\right)+\left(1+v^{2}\right)\left(t^{2}+x^{2}\right)\right. \\
& \left.-2 t\left(\left(1+v^{2}\right) \varepsilon+2 v x\right)+2(1+(2-v) v) x \varepsilon+2 \varepsilon^{2}\right] .
\end{aligned}
$$

We do not write $u_{3 C}(t, x)$ because its expression is too complicated to be analytically obtained. However, we can use the finite differences method to take the derivatives of $\phi_{3 C}(t, x)$ necessary to calculate its energy density.

The expressions (4.11)-(4.15) and the numerical data for $u_{3 C}(t, x)$ were used to plot the energy density as a color gradient for $0 \leq t \leq t_{1}$ (Fig. 19, right-hand side).

Looking closely to Fig. 19 we can see the energy density getting dimmer as the solution evolves in time. Such an effect is particularly visible for larger values of $\epsilon$ and gets less noticeable as $\epsilon$ decreases. This behavior suggests that the outer regions of the solution act as a reservoir of energy, feeding the expansion of the inner structures (shocklike wave).

The partial solutions $\phi_{2 C}$ and $\phi_{3 C}$ correspond to partial solutions of the exact shockwave. Therefore, $\phi_{C}, \phi_{1 L}$, $\phi_{2 L}, \phi_{3 L}$ (and the corresponding right-side solutions) are related to our approximation of the Dirac delta by a finite function. When $\epsilon \rightarrow 0$, these solutions reduce to a discontinuity in the field. The color gradient plots suggests that these partial solutions loose energy over time. We can examine this claim closer by integrating the energy densities and obtain the total energy as a function of time:

$$
\begin{gathered}
E_{1 C}(t)= \begin{cases}\frac{t\left(2 t^{2}(v+3)-6 t \varepsilon+3 \varepsilon^{2}\right)}{3(v+1)^{2}} & \text { if } 0 \leq t \leq \frac{\varepsilon}{2} \\
\frac{(\varepsilon-t)\left(2 t^{2}(v+3)+2 t(v-3) \varepsilon-(v-3) \varepsilon^{2}\right)}{3(v+1)^{2}} & \text { if } \frac{\varepsilon}{2}<t \leq \varepsilon\end{cases} \\
E_{1 L}(t)=E_{1 R}(t)=\frac{-8 t^{3}+6 t^{2} \varepsilon-3 t \varepsilon^{2}+\varepsilon^{3}}{6(v+1)^{2}}
\end{gathered}
$$




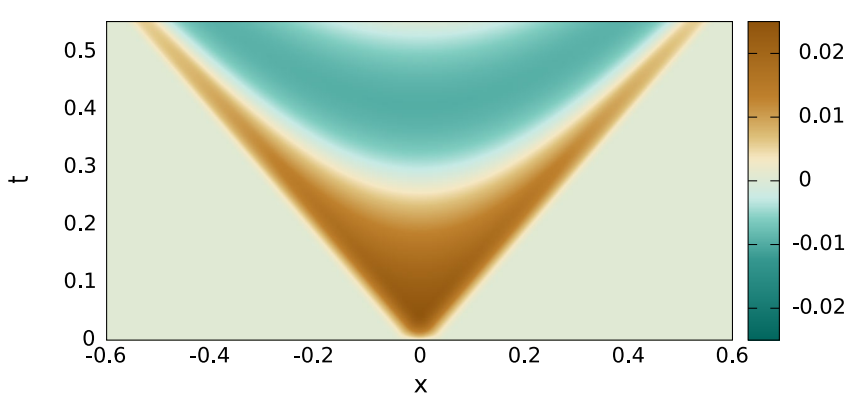

(a) $\epsilon=2 \cdot 10^{-4}$

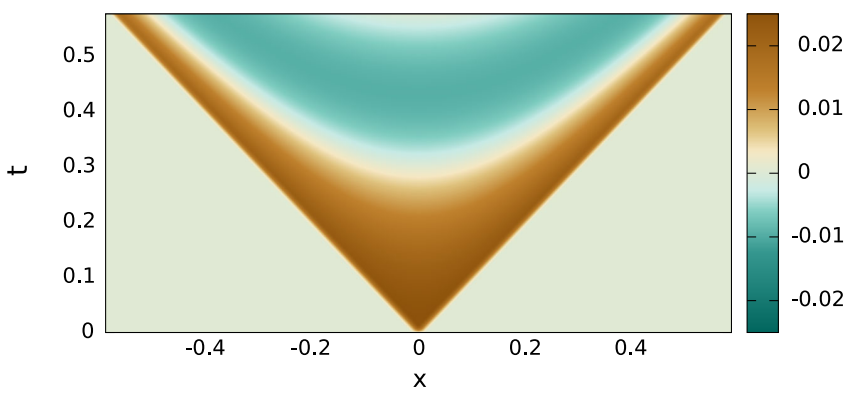

(c) $\epsilon=2 \cdot 10^{-5}$

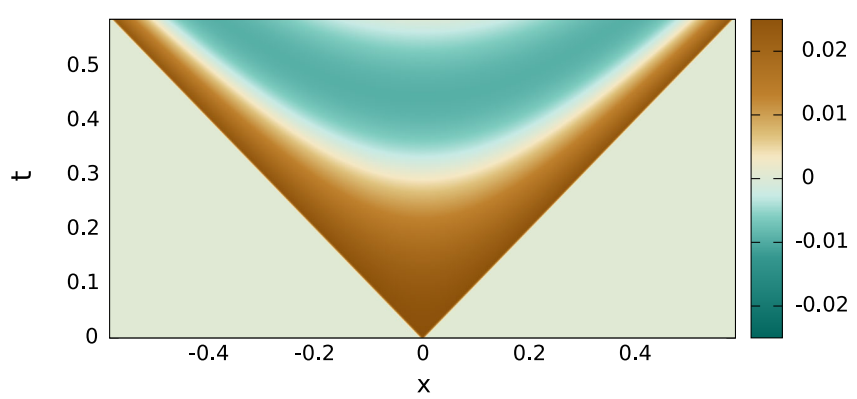

(e) $\epsilon=2 \cdot 10^{-6}$

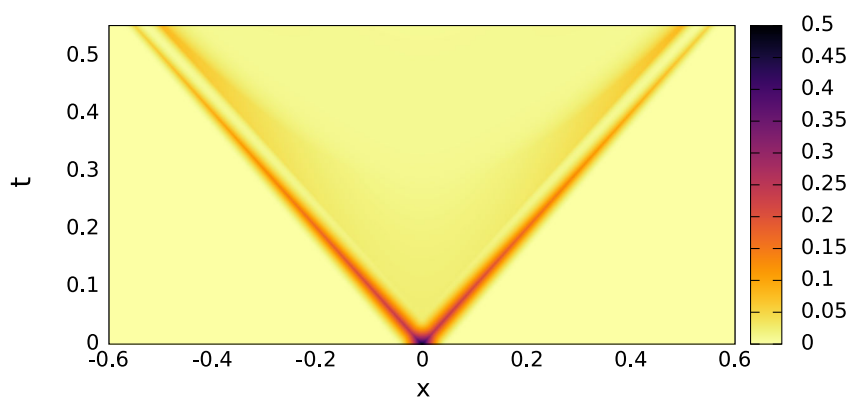

(b) $\epsilon=2 \cdot 10^{-4}$

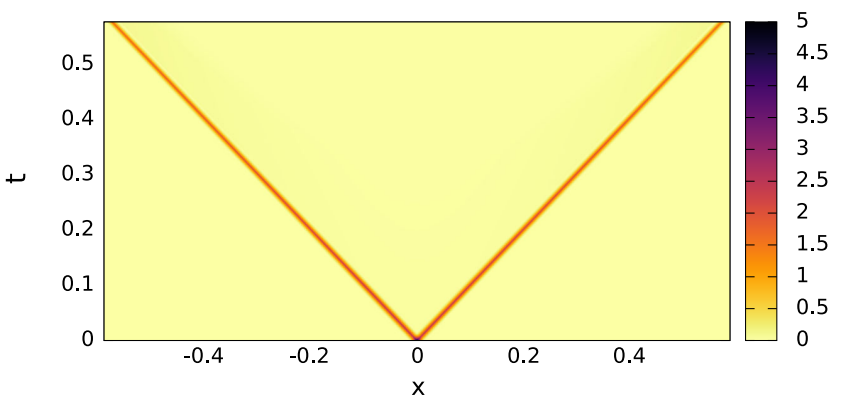

(d) $\epsilon=2 \cdot 10^{-5}$

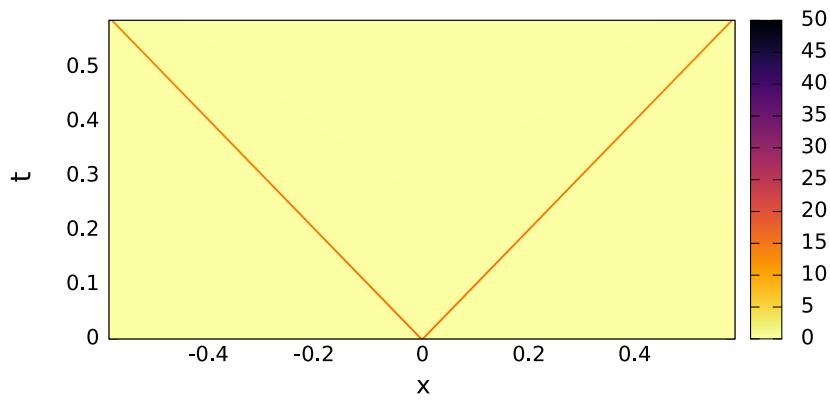

(f) $\epsilon=2 \cdot 10^{-6}$

FIG. 19. Field $\phi(t, x)$ (left) and its energy density (right) for $a=0.05$.

$$
\begin{gathered}
E_{2 L}(t)=E_{2 R}(t)= \begin{cases}-\frac{t^{3}(v-1)}{3(v+1)^{2}} & \text { if } 0 \leq t \leq \frac{\varepsilon}{2} \\
-\frac{((1+v) t-\varepsilon)^{3}}{3\left(v^{2}-1\right)^{2}} & \text { if } t>\frac{\varepsilon}{2}\end{cases} \\
E_{3 L}(t)=E_{3 R}(t)= \begin{cases}\frac{(2 t-\varepsilon)\left(2 t^{2}[v(v(v+2)-1)+2]+t[v(v(v-7)+5)-7] \varepsilon-[v(v(v-4)+5)-4] \varepsilon^{2}\right)}{6\left(v^{2}-1\right)^{2}} & \text { if } \frac{\varepsilon}{2} \leq t \leq \varepsilon \\
\frac{\varepsilon\left(6 t^{2}(v+1)^{2}+3 t[v(v-2)-5](v+1) \varepsilon-[v(v(v+2)-7)-10] \varepsilon^{2}\right)}{6\left(v^{2}-1\right)^{2}} & \text { if } t>\varepsilon .\end{cases}
\end{gathered}
$$

Considering the intervals of time where each solution is valid, we can sum up the energy contained in the outer regions of the field. That is, the energy contained in the regions that are reduced to discontinuities when $\epsilon$ tends to zero.

Looking at Fig. 20 we see that the energy in the outer regions is initially constant 


$$
\begin{aligned}
& E_{1 C}(t)+E_{1 L}(t)+E_{1 R}(t)+E_{2 L}(t)+E_{2 R}(t)=\frac{a^{2}}{3 \varepsilon}=\frac{a^{2}}{6 \sqrt{\pi \epsilon}}, \quad \text { if } 0 \leq t \leq \frac{\varepsilon}{2}, \\
& E_{1 C}(t)+E_{3 L}(t)+E_{3 R}(t)+E_{2 L}(t)+E_{2 R}(t)=\frac{a^{2}}{3 \varepsilon}=\frac{a^{2}}{6 \sqrt{\pi \epsilon}}, \quad \text { if } \frac{\varepsilon}{2}<t \leq \varepsilon,
\end{aligned}
$$

and later it decreases approximately linearly for small $\varepsilon$. This decreasing of energy originates with the appearance of the inner solution $\phi_{2 C}$ and then $\phi_{3 C}$. It can be expected that when the energy gets sufficiently small, it can no longer feed the expansion of the shockwave. Therefore the shockwave breaks down and radiates.

\section{Shock waves and scattering of oscillons}

One of the most characteristic properties of a radiation generated from initial data (4.1) is an amazing similarity between their patterns and the patterns formed by radiation released during the process of scattering of oscillons. The problem of scattering of oscillons has been reported in Ref. [18]. The initial configuration contains two exact oscillons with compact supports that touch each other at $t=0$. Such oscillons move in front of each other with equal speeds in the laboratory reference frame. In order to simplify considerations we take only the initial configurations which are symmetric under spatial reflections $x \rightarrow-x$. The scattering process leads to the emergence of two main oscillons and production of the radiation (smaller oscillons waves, etc.). This radiation is mainly concentrated in the central region of the spacetime diagram. There are two parameters which we use to get different initial configurations: speeds of initial oscillons $V$ and their phase $\alpha$. Dependently on the value of these parameters (on the form of the initial oscillons) we get a variety of different patterns of the radiation.

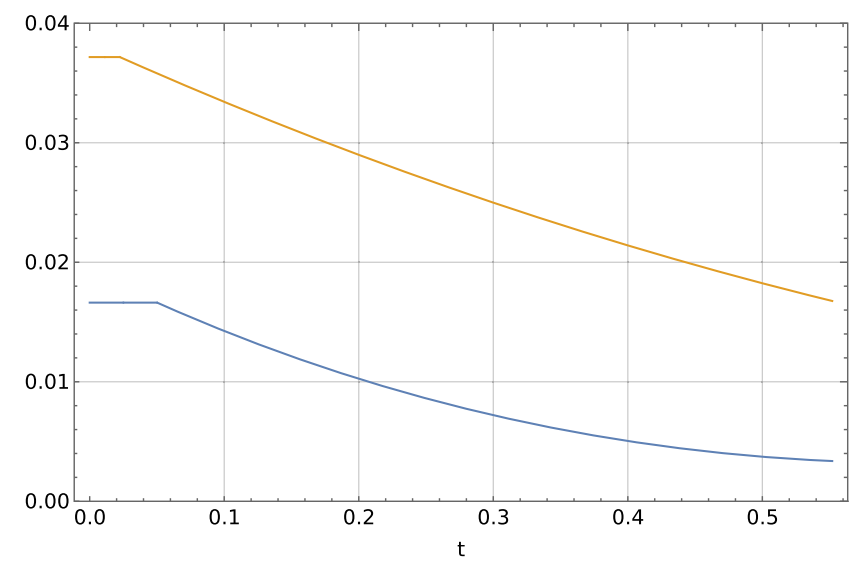

FIG. 20. Total energy carried by the partial solutions $\phi_{1 C}, \phi_{1 L}$, $\phi_{1 R}, \phi_{2 L}, \phi_{2 R}, \phi_{3 L}$, and $\phi_{3 R}$ for $\epsilon=2 \times 10^{-4}$ (bottom) and $\epsilon=$ $4 \times 10^{-5}$ (top).
In Fig. 21 we show three examples of the evolution of the signum-Gordon field. The magnitude of the field is represented by a gradient color. Subfigures (a), (c), (e) show the scattering of two oscillons in dependence on their initial speed $V$ and the phase $\alpha$, whereas subfigures (b), (d), (f) show the evolution of some initial field configurations given by (4.1) (Gaussian case) with different values of $\epsilon$. The left and right figures look amazingly similar taking into account that there is no direct relation between their initial conditions. The presence of a radiation in the scattering process means that the two emerging (leading) oscillons have less energy than the incoming ones. The difference of the energy is carried by the radiation. Having in mind that the appearance of a shock wave solution requires a deltalike initial field configuration we can speculate that such a configuration of the field could be produced shortly after collision (at the moment when two main outgoing oscillons arise). This field configuration would give rise to the shock wave solution in the similar way as the initial data (4.1) does. In this scenario the role of a scattering process is limited to the generation of a field configuration which furthermore develops a shock-wave-like form. It explains to some extent the universal character of the patterns formed by radiation of the signum-Gordon field.

\section{CONCLUSIONS}

We have presented some considerations concerning the problem of a collapse of shocklike wave solutions in the signum-Gordon model. This phenomenon was observed previously in the collision between two oscillons. Since the oscillons dominate the radiation spectrum of the model and they collide frequently the decay of shock waves is an efficient mechanism of production of small size oscillons.

In the first part of the paper we looked in more detail at the exact shock wave solution. We manage to establish the initial condition for exact shock waves. Namely, a suitable initial field configuration contains the field which vanishes everywhere and its time derivative is proportional to the Dirac delta. The support of the wave is localized inside the light cone including the cone. We have shown that the energy of the solution inside the light cone (excluding the cone) increases linearly with time. This result may look strange when confronted with the fact that the signumGordon model conserves the energy. In fact there is no inconsistency between these two facts. The total energy of the shock waves includes also the gradient energy 


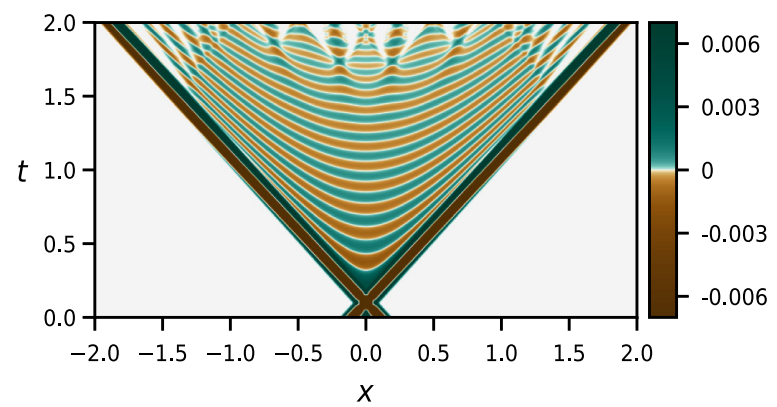

(a)

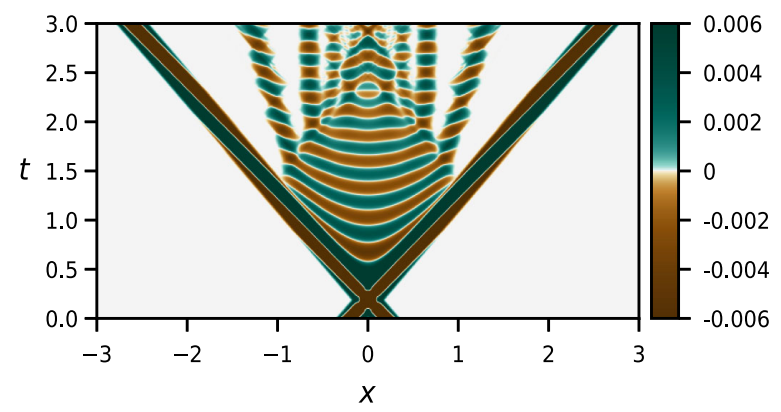

(c)

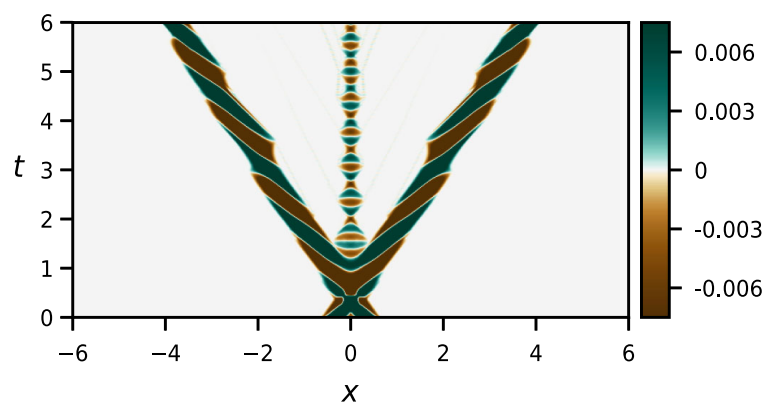

(e)

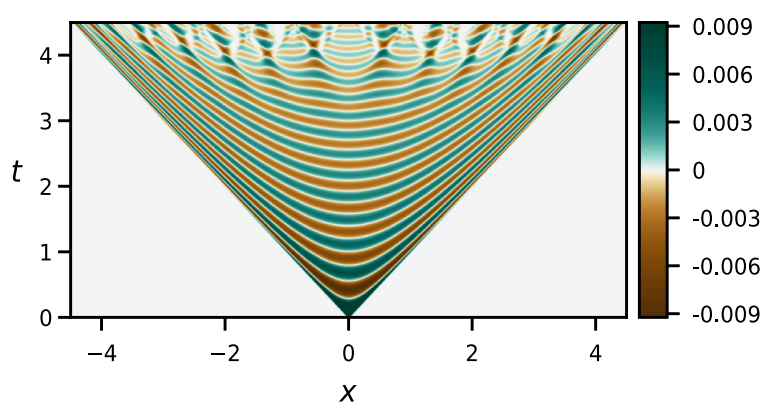

(b)

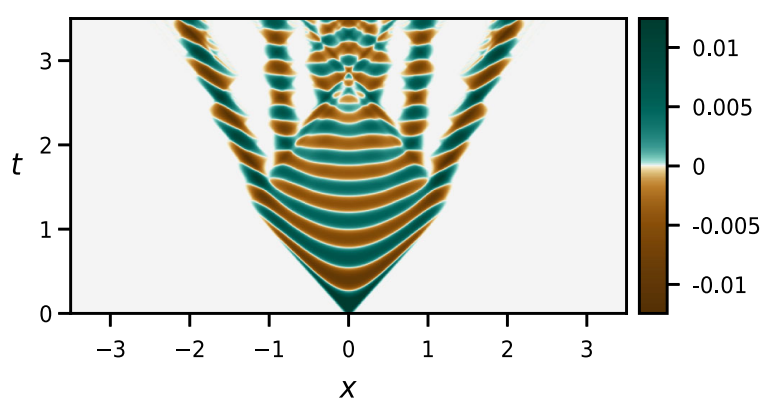

(d)

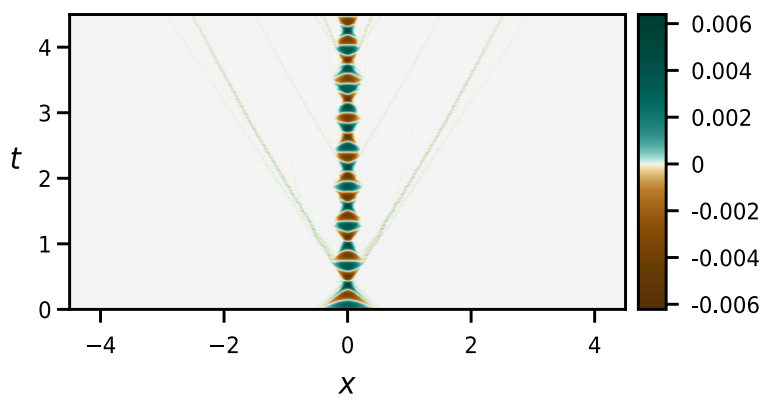

(f)

FIG. 21. Scattering of symmetric oscillons (left) and evolution of initial data (4.1) (right). The parameters of subfigures (a) $(V, \alpha)=(0.98,0.648), \quad(b) \quad(a, \epsilon)=\left(0.005,1.747 \times 10^{-5}\right), \quad(\mathrm{c}) \quad(V, \alpha)=(0.93,0.680), \quad(\mathrm{d}) \quad(a, \epsilon)=\left(0.005,2.288 \times 10^{-4}\right)$, (e) $(V, \alpha)=(0.74,0.089)$, and (f) $(a, \epsilon)=\left(0.005,3.0959 \times 10^{-2}\right)$.

associated with discontinuities of the field at the light cone. This energy is clearly infinite. Thus the existence of the exact shock waves requires a continuous transfer of the energy from discontinuities to the region inside the light cone.

Next we have looked at the systems with finite total energy. Taking the regular $\delta$-like initial profile of $\partial_{t} \phi$ we got a numerical solution which initially looks very similar to exact shock wave solution. The finiteness of the gradient energy of the field close to the light cone did not allow for the existence of the wave for arbitrary long times. We found that the wave begins to collapse (decay) into oscillons. The decay starts earlier for configurations with lower the gradient energy (bigger $\epsilon$ ).
The numerical solutions obtained for initial configurations that differ by a value of $\epsilon$ are very similar to solutions obtained in the process of scattering of exact oscillons. Our analysis of shock waves allows for a better understanding of how oscillons are produced in the collision of two incoming oscillons: the outgoing oscillons have less energy than the incoming ones which leads to the production of waves that eventually decay into oscillons.

The observed way of production of oscillons in a decay of shocklike wave solution is also expected in other models with V-shaped potentials in the limit of small amplitudes of the field. Our preliminary results from the scattering of compact kinks in double-well potential confirm this statement. 


\section{ACKNOWLEDGMENTS}

The authors would like to thank H. Arodź, A. Wereszczyński, and W. J. Zakrzewski for discussions and comments. F. M. H. is supported by a CNPq Scholarship
(CNPq Scholarship holder-Brazil) and J. S. S. by a CAPES Scholarship. This study was financed in part by the Coordenação de Aperfeiçoamento de Pessoal de Nível Superior-Brasil (CAPES)—Finance Code 001.
[1] H. Arodź, P. Klimas, and T. Tyranowski, Compact oscillons in the signum-Gordon model, Phys. Rev. D 77, 047701 (2008).

[2] H. Arodź, P. Klimas, and T. Tyranowski, Field-theoretic models with V-shaped potentials, Acta Phys. Pol. B 36, 3861 (2005).

[3] I. L. Bogolyubsky and V. G. Makhankov, On the lifetime of pulsing solitons in some classical models, Pis'ma Zh. Éksp. Teor. Fiz. 24, 15 (1976).

[4] M. Gleiser, Pseudostable bubbles, Phys. Rev. D 49, 2978 (1994).

[5] M. Gleiser, Oscillons in scalar field theories: Applications in higher dimensions and inflation, Int. J. Mod. Phys. D 16, 219 (2007).

[6] T. Romanczukiewicz and Ya. Shnir, Oscillon Resonances and Creation of Kinks in Particle Collisions, Phys. Rev. Lett. 105, 081601 (2010).

[7] Tomasz Romańczukiewicz and Yakov Shnir, Oscillons in the presence of external potential, J. High Energy Phys. 01 (2018) 101.

[8] R. A. C. Correa, W. de Paula, T. Frederico, O. Oliveira, and F. E. M. Silveira, Oscillons in $\phi^{6}$-theories: Possible occurrence in MHD, arXiv:1806.04412.

[9] M. J. Ablowitz, D. J. Kaup, A. C. Newell, and H. Segur, Method for Solving the Sine-Gordon Equation, Phys. Rev. Lett. 30, 1262 (1973).

[10] M. J. Ablowitz, D. J. Kaup, A. C. Newell, and H. Segur, Nonlinear-Evolution Equations of Physical Significance, Phys. Rev. Lett. 31, 125 (1973).

[11] D. I. Olive, N. Turok, and J. W. R. Underwood, Affine Toda solitons and vertex operators, Nucl. Phys. B409, 509 (1993).

[12] L. A. Ferreira and W. J. Zakrzewski, A simple formula for the conserved charges of soliton theories, J. High Energy Phys. 09 (2007) 015.
[13] P. Klimas, J. S. Streibel, A. Wereszczyński, and W. J. Zakrzewski, Oscillons in a perturbed signum-Gordon model, J. High Energy Phys. 04 (2018) 102.

[14] C. Adam, D. Foster, S. Krusch, and A. Wereszczyński, BPS sectors of the Skyrme model and their non-BPS extensions, Phys. Rev. D 97, 036002 (2018).

[15] C. Adam, P. Klimas, J. Sanchez-Guillen, and A. Wereszczyński, Compact baby Skyrmions, Phys. Rev. D 80, 105013 (2009).

[16] P. Klimas and L. R. Livramento, Compact Q-balls and Q-shells in $C P^{N}$-type models, Phys. Rev. D 96, 016001 (2017).

[17] Y. M. Shnir, Topological and Non-Topological Solitons in Scalar Field Theories (Cambridge University Press, Cambridge, England, 2018).

[18] F. M. Hahne, P. Klimas, J. S. Streibel, and W. J. Zakrzewski, Scattering of compact oscillons, J. High Energy Phys. 01 (2020) 006.

[19] C. Adam, J. Sanchez-Guillen, and A. Wereszczyński, A Skyrme-type proposal for Baryonic matter, Phys. Lett. B 691, 105 (2010).

[20] H. Arodz, P. Klimas, and T. Tyranowski, Scaling, selfsimilar solutions and shock waves for V-shaped field potentials, Phys. Rev. E 73, 046609 (2006).

[21] H. Arodz, P. Klimas, and T. Tyranowski, Signum-Gordon wave equation and its self-similar solutions, Acta Phys. Pol. B 38, 3099 (2007).

[22] H. Arodz and Z. Świerczyński, Swaying oscillons in the signum-Gordon model, Phys. Rev. D 84, 067701 (2011).

[23] Z. Świerczyński, On the oscillons in the signum-Gordon model, J. Nonlinear Math. Phys. 24, 20 (2017).

[24] P. Klimas, On shock waves in models with V-shaped potentials, Acta Phys. Pol. B 38, 21 (2007). 